\title{
Effects of a 16-week multimodal exercise program on gait performance in individuals with dementia: a multicenter randomized controlled trial
}

Sandra Trautwein ${ }^{1 *}$ D, Bettina Barisch-Fritz ${ }^{1}$, Andrea Scharpf ${ }^{1}$, Steffen Ringhof ${ }^{1,2}$, Thorsten Stein ${ }^{1}$, Janina Krell-Roesch ${ }^{1,3}$ and Alexander Woll ${ }^{1}$

\begin{abstract}
Background: There is a high prevalence of gait impairments in individuals with dementia (IWD). Gait impairments are associated with increased risk of falls, disability, and economic burden for health care systems. Only few studies have investigated the effectiveness of physical activity on gait performance in IWD, reporting promising but inconsistent results. Thus, this study aimed to investigate the effectiveness of a multimodal exercise program (MEP) on gait performance in IWD.

Methods: In this parallel-group randomized controlled trial, we enrolled 319 IWD of mild to moderate severity, living in care facilities, aged $\geq 65$ years, and being able to walk at least $10 \mathrm{~m}$. The control group $(n=118)$ received conventional treatment, whereas the intervention group $(n=201)$ additionally participated in a 16-week MEP specifically tailored to IWD. We examined the effects of the MEP on spatiotemporal gait parameters and dual task costs by using the gait analysis system GAITRite. Additionally, we compared characteristics between positive, non-, and negative responders, and investigated the impact of changes in underlying motor and cognitive performance in the intervention group by conducting multiple regression analyses.

Results: Two-factor analyses of variance with repeated measurements did not reveal any statistically significant time* group effects on either spatiotemporal gait parameters or dual task costs. Differences in baseline gait performance, mobility, lower limb strength, and severity of cognitive impairments were observed between positive, non-, and negative responders. Positive responders were characterized by lower motor performance compared to negative and non-responders, while non-responders showed better cognitive performance than negative responders. Changes in lower limb strength and function, mobility, executive function, attention, and working memory explained up to $39.4 \%$ of the variance of changes in gait performance.

(Continued on next page)
\end{abstract}

* Correspondence: sandra.trautwein@kitedu

'Karlsruhe Institute of Technology, Institute of Sports and Sports Science, Engler-Bunte-Ring 15, 76131 Karlsruhe, Germany

Full list of author information is available at the end of the article

C C The Author(s). 2020 Open Access This article is licensed under a Creative Commons Attribution 4.0 International License, which permits use, sharing, adaptation, distribution and reproduction in any medium or format, as long as you give appropriate credit to the original author(s) and the source, provide a link to the Creative Commons licence, and indicate if changes were made. The images or other third party material in this article are included in the article's Creative Commons licence, unless indicated otherwise in a credit line to the material. If material is not included in the article's Creative Commons licence and your intended use is not permitted by statutory regulation or exceeds the permitted use, you will need to obtain permission directly from the copyright holder. To view a copy of this licence, visit http://creativecommons.org/licenses/by/4.0/. The Creative Commons Public Domain Dedication waiver (http://creativecommons.org/publicdomain/zero/1.0/) applies to the data made available in this article, unless otherwise stated in a credit line to the data. 
(Continued from previous page)

Conclusions: The effectiveness of a standardized MEP on gait performance in IWD was limited, probably due to insufficient intensity and amount of specific walking tasks as well as the large heterogeneity of the sample. However, additional analyses revealed prerequisites of individual characteristics and impacts of changes in underlying motor and cognitive performance. Considering such factors may improve the effectiveness of a physical activity intervention among IWD.

Trial registration: DRKS00010538 (German Clinical Trial Register, date of registration: 01 June 2016, retrospectively registered, https://www.drks.de/drks_web/setLocale_EN.do).

Keywords: Physical activity, Neurodegenerative disorder, Walking, Physical functional performance, Cognition, Dual task

\section{Background}

Gait impairments represent a major public health concern [1]. Their prevalence increases with age, and more than $32 \%$ of individuals aged 60 years and above have gait impairments [2] such as decreased walking speed, shortened stride length, and enhanced double support phase $[1,3,4]$. Gait impairments are very prevalent in individuals with dementia (IWD), with an estimated 50\% of IWD being affected $[5,6]$. In contrast, among cognitively unimpaired older adults, the prevalence rates range between 7 and $36 \%[2,5,6]$. Dementia is an umbrella term for conditions that are characterized by a significant decline in one or more cognitive domains and behavioral changes that interfere with independence in everyday activities [7]. The causes of dementia can vary, with Alzheimer's disease being the most common in older adults. Other dementias include vascular dementia, Lewy body dementia, or frontotemporal dementia. Furthermore, mixed dementia is common [8].

Various other motor impairments, such as reduced strength and postural control, may contribute to the increased prevalence of gait impairments in IWD $[5,9]$. Moreover, gait is not merely an automated motor activity but requires input from the cerebellum, the motor cortex, and the basal ganglia, as well as an intact sensory feedback $[1,10,11]$. Thus, dementia-related pathological changes in these brain structures may also contribute to gait impairments [3].

Both gait and cognitive impairments are associated with an increased risk of falls and decreased quality of life [12, 13]. Accordingly, the incidence of falls in IWD is two to three times higher than in cognitively unimpaired older individuals $[1,5,13]$. Furthermore, the various healthrelated and economic consequences of falls, such as higher rates of institutionalization, disability, morbidity, mortality, and increased financial burden $[1,5]$, underline the need of interventions focusing on improving or maintaining gait performance in IWD. Indeed, various pharmacological and non-pharmacological interventions to improve gait performance and reduce falls in older adults have been studied $[1,5,11,13-16]$.
Physical activity interventions have shown to be effective in cognitively unimpaired older individuals and may also be beneficial for IWD [5]. However, to date, only few studies have evaluated the effectiveness of physical activity on gait performance in IWD. These studies show promising but inconsistent results. For example, seven studies observed positive effects of physical activity on walking speed as assessed through short distance walk tests [17-23], whereas fifteen studies did not report statistically significant findings [24-38]. Furthermore, ten studies applied an instrumented gait analysis, and mainly reported positive effects of physical activity on stride length [39-46], stride time [43, 45], step time [46], double support time [40], and stride frequency [41]. In contrast, no effects were found on step length $[44,47]$, step width $[43,47]$, and percent of single support [44]. Inconsistent results exist for walking speed [40-44, 46-48], stride speed [39, 45], percent of double support [43-45], and cadence [19, 39, 42, $43,45,46]$. Findings of studies investigating dual task conditions are also inconsistent regarding potential effects on gait impairments and do not allow meaningful conclusions [26, 28, 35, 36, 39, 45]. Thus, more research is needed to better understand the potentially beneficial effects of physical activity on gait performance in IWD in both single and dual task conditions.

Most previous studies conducted multimodal physical activity interventions, including strength, balance, and aerobic exercises [17-21, 23-25, 28, 30-36, 39-45, 47]. Given the relationship between motor, cognitive and gait performance, as well as the positive impacts of cognitive training programs $[1,11]$, interventions combining physical and cognitive activity may be most promising for improving gait performance in IWD [15]. Indeed, studies combining physical and cognitive activity predominantly reported beneficial effects on gait performance [35, 39, $43,45]$. However, these studies had no randomized controlled trial designs $[35,39,45]$, did not use instrumented gait analysis systems [35], or focused on dual task exercises while not considering other cognitive tasks [43]. This research gap emphasizes the need for additional investigations. 
When aiming to improve the effectiveness of physical activity interventions on gait performance in IWD, it is also important to consider and identify determinants that may potentially impact the association between physical activity and subsequent changes in gait performance. However, research on such prerequisites, e.g. specific characteristics of participants that may determine which participants are most likely to benefit from specific physical activity interventions, is rare. With regard to the expected direct and indirect effects of physical activity (see Fig. 1), little is known as to how intervention-induced changes in underlying motor and cognitive performance may be related to changes in gait performance in IWD. As both other motor and cognitive impairments explain the increased prevalence of gait impairments in IWD [5], potential impacts of both factors are possible. Based on theoretical considerations, associations between changes in gait performance with changes in balance, mobility, strength and function of lower limbs [9] as well as with changes in executive function, attention, and working memory $[49,50]$ can be assumed.

Therefore, the primary aim of the current study was to determine the effectiveness of a multimodal exercise program (MEP), which combined both motor and cognitive tasks, on gait performance and dual task costs in IWD residing in care facilities. We hypothesized that a 16-week MEP, in addition to conventional treatment, may have a differential effect on gait performance in IWD as compared to conventional treatment alone. Our secondary aim was to identify determinants that may affect the effectiveness of the MEP, by examining differences in characteristics closely related to gait performance between positive, non-, and negative responders. Based on relations of gait performance with motor and cognitive performance, as well as the etiology of dementia $[1,3,5,9-11]$, we hypothesized that positive, non-, and negative responders of the MEP would differ in baseline performance (i.e. gait, balance, mobility, strength and function of lower limbs, executive function, attention, and working memory) and selected sample characteristics (i.e. severity of cognitive impairments, etiology of dementia, and use of walking aids). Furthermore, we also investigated impacts of interventioninduced changes in underlying motor and cognitive performance on changes in gait performance and hypothesized that changes in underlying motor (balance, mobility, strength and function of lower limbs) and cognitive performance (executive function, attention, and working memory) may have an impact on changes in gait performance in IWD who participated in a dementia-specific MEP. Due to limited prior research related to our secondary and third aim, we examined these research questions based on an exploratory analysis approach.

\section{Methods}

For this manuscript, we followed the guidelines and recommendations of the Consolidated Standards of Reporting Trials statements [51, 52]. The reader is referred to the published study protocol for a detailed description of the study design and methods [53]. The following sections will only provide a brief summary of study methods. Further information is available in the German National Register of Clinical Trials (DRKS00010538), where we retrospectively registered this study. The ethics committee of the Karlsruhe Institute of Technology (Karlsruhe, Germany) granted ethical approval.

\section{Study design}

We performed a multicenter, parallel group randomized controlled trial with baseline and post-intervention assessments. We allocated participants to an intervention group (IG) or control group (CG) with an allocation ratio of 2:1 using minimization software (MinimPy, Version 0.3 [54]). This allocation ratio accounts for assumed higher dropout quotes and insufficient adherence to the MEP in the IG. The randomization was carried out separately for each of the 36 care facilities that served as recruitment sites, and minimization considered age, sex, Mini-Mental State Examination (MMSE) score and performance in the modified 30-s chair stand test (30s CST) of participants

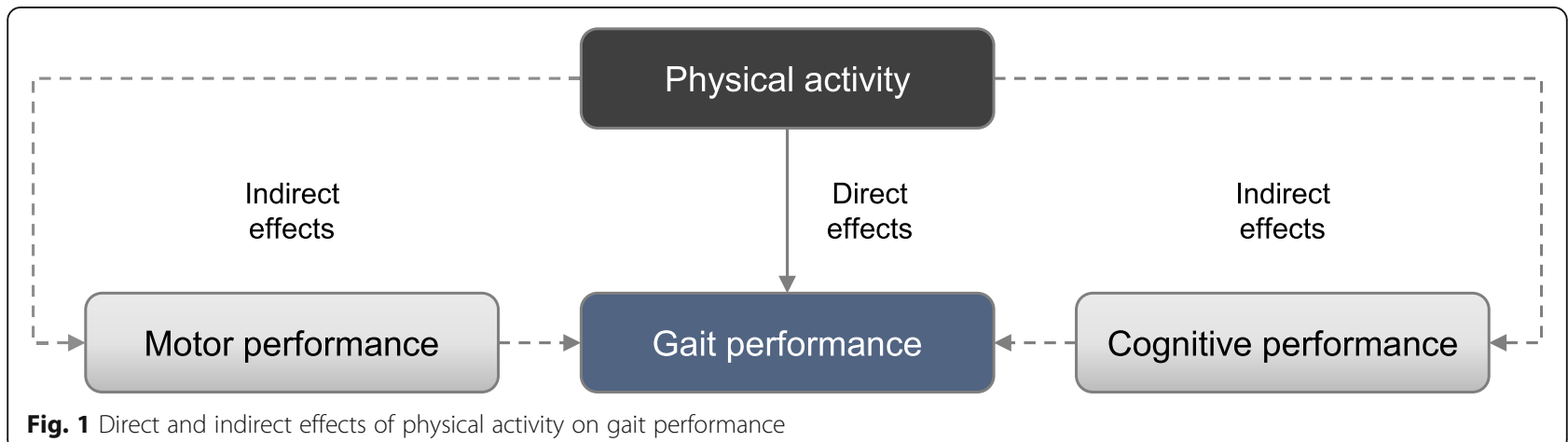

Fig. 1 Direct and indirect effects of physical activity on gait performance 
(for details please refer to our study protocol [53]). If possible, we blinded investigators who examined gait, motor, and cognitive performance to group allocation, i.e., the investigators did not know whether a participant was part of the IG or CG in order to ensure objectivity of testing. However, it was not possible to blind participants. In few cases, participants themselves disclosed their group allocation to investigators.

\section{Participants}

A power analysis (G*Power 3, Version 3.1.9.2 [55], twofactor analysis of variance (ANOVA) with repeated measurements, two groups, two measurements, $\alpha=0.05$, 1 $\beta=0.80, \eta^{2}=0.01$ ) revealed a required total sample size of 200 participants. Considering various potential reasons for dropout, missing data, and low adherence to the MEP, we set the sample size to 405 participants. Participants were recruited from 36 care facilities in SouthWestern Germany that had been contacted by the research team and asked whether they would be interested to participate in the study. Employees of the respective care facilities that were identified by the respective leadership to help with the conduct of the study identified potential eligible participants, which had to fulfill the following inclusion and exclusion criteria:

Inclusion criteria: a) diagnosis of dementia or "suspected" dementia (i.e. person with dementia as suspected by the treating physician based on ICD-10 criteria and MMSE performance but without a confirmed diagnosis or awaiting further clinical evaluation); b) Alzheimer's disease, vascular dementia, or other primary dementia; c) mild to moderate severity of dementia (MMSE score: 10-24); d) age above 65 years; e) walking ability of about 10 meters with or without walking aid; and f) clearance from general practitioner.

Exclusion criteria: a) secondary dementia; b) other severe cognitive impairments; $c$ ) other severe neurological diseases; d) any severe acute diseases; and e) severe motor impairments.

Based on these inclusion and exclusion criteria, we verified the eligibility of participants at baseline assessment. Furthermore, we obtained written informed consent prior to the study from all participants or their legal guardians, respectively. Participation in this study was voluntary.

\section{Sample characteristics}

The employees of the care facilities documented characteristics of participants including sex, year of birth, diagnosis of dementia, etiology of dementia, walking aids, depression, Cumulative Illness Rating Scale (CIRS) [56], and medication intake within 2 weeks of baseline assessments based on medical reports. Whenever possible, we asked physicians to retrospectively provide any missing information on their patients. In addition, we measured body weight and height in all participants.

\section{Intervention}

Details of the intervention including examples of motor and cognitive tasks, ritualization as well as progression of exercise intensity and requirements during the course of the study are described in the study protocol [53]. Briefly, participants in the IG underwent an MEP combining motor (i.e. strength, balance, endurance, and flexibility) and cognitive tasks (i.e. memory, attention, language, and executive function). The MEP was tailored to fit the specific needs (e.g. sense of security and safety, clear verbal instruction, simplicity of tasks) and characteristics (e.g. impaired memory and attention, decreased mobility, increased fall risk) of IWD, and was delivered in the care facilities by instructors who had been specifically trained for the purpose of this study. All instructors were experienced in sports science and participated in a comprehensive training focusing on structure and contents of MEP as well as specific demands of IWD prior to delivering the intervention.

In order to provide a sense of security for participants, the MEP included a ritualization that ensured an identical sequence for all sessions. During each session, participants were asked to imagine a journey while performing appropriate motor and cognitive exercises. For example, participants performed different mobilization exercises while imagining packing their luggage as beginning ritual. The journey as well as related motor and cognitive exercises alternated throughout the course of the study. The MEP took place twice a week over a period of 16 weeks. Sessions had a duration of $60 \mathrm{~min}$ including about 45 min of physical exercise (the remaining $15 \mathrm{~min}$ were used to explain exercises, to welcome and say goodbye to participants, to answer questions or to take a short break and drink water). The MEP was delivered in a group setting (max. 12 participants, 2 instructors) and was mainly performed in a seated position with medium to submaximal intensity. Furthermore, it contained tasks in standing position and specific walking exercises. Intensity and requirements were determined on the basis of duration of exercises, number of repetitions, applied training devices, and amount of tasks in standing position as well as walking exercises. During the course of the 16 weeks, we increased the intensity of the sessions as well as the degree of motor and cognitive requirements, which were determined in the training manual. Both CG and IG participants received individually tailored conventional treatment (e.g. medication, care, or therapeutic applications) as part of standard care in their care facilities. 


\section{Outcome measurements}

We examined gait performance as outcome of interest with various spatiotemporal gait parameters of the right leg: walking speed $(\mathrm{m} / \mathrm{sec})$, stride length $(\mathrm{cm})$, stride time (sec), double support phase (\% of stride time), and stance phase (\% of stride time). Gait analysis was performed using the electronic gait analysis system GAITRite (CIR Systems Inc., Franklin, USA, active length of $4.88 \mathrm{~m}$ ), which has been shown to be reliable in IWD [57, 58]. All participants underwent gait analysis in single and two dual task conditions (i.e. counting backwards starting from 50 and naming animals while walking) to also assess dual tasks costs of walking while talking $[59,60]$. The same dual tasks were applied for baseline and post assessment.

To eliminate acceleration and deceleration during the recording, we asked participants to start walking two meters in front of the GAITRite system and to stop walking two meters behind the system [59]. While walking at comfortable speed, participants were allowed to use walking aids as applied in everyday life. Instructions were repeated if necessary. We asked participants to repeat all conditions up to five times to generate three valid walks. Valid trials consisted of a minimum of six consecutive steps of steady-state walking, and complied with satisfactory cognitive performance in dual task conditions (i.e., stating at least three numbers in correct order and naming at least one animal, respectively). To prevent potential bias due to fatigue, participants were asked to rest between the trials if needed and we limited the assessment to five trials; thus, few participants had less than three valid trials. For statistical analysis, we considered the trial with the smallest difference to mean walking speed of all valid trials of one condition. We calculated dual-task costs using the equation $\frac{\text { dual task - single task }}{\text { single task }} \times 100[61,62]$. Dual task costs show the percentage difference between parameters assessed during single and dual task conditions (e.g., walking speed or stride length) and thus reflect the impact of the additional cognitive task on these spatiotemporal gait parameters $[61,62]$.

In order to analyze differences between positive, negative, and non-responders, as well as impacts of changes in underlying motor and cognitive performance on changes in gait performance, we determined related outcomes using the motor and cognitive assessments displayed in Table 1. Experienced study staff with background in sports science supervised and administered all assessments. The reader is referred to the published study protocol [53] for a detailed description of all assessments.

\section{Statistical analysis}

Statistical analysis was performed using IBM SPSS Version 25 (IBM Corporation, Armonk, USA). We ran a per
Table 1 Motor and cognitive assessments to analyze differences between positive, non-, and negative responders, as well as impacts of changes in underlying motor and cognitive performance on changes in gait performance

\begin{tabular}{|c|c|}
\hline Outcome & Assessment \\
\hline Balance & $\begin{array}{l}\text { Frailty and Injuries: Cooperative Studies of } \\
\text { Intervention Techniques - subtest } 4 \text { (score) } \\
\text { [63] }\end{array}$ \\
\hline Mobility & Timed Up \& Go Test (TUG; time in s) [64] \\
\hline $\begin{array}{l}\text { Strength and function of } \\
\text { lower limb }\end{array}$ & $\begin{array}{l}\text { Modified 30-Second Chair-Stand Test (30s } \\
\text { CST; number of repetitions) }[65,66] \\
\text { Modified Short Physical Performance } \\
\text { Battery (SPPB; score) }[67]^{a}\end{array}$ \\
\hline Global cognition & $\begin{array}{l}\text { Mini-Mental State Examination (MMSE; } \\
\text { score) [68] }\end{array}$ \\
\hline $\begin{array}{l}\text { Executive function and } \\
\text { visual-spatial cognition }\end{array}$ & $\begin{array}{l}\text { Clock Drawing Test (adapted Sunderland } \\
\text { score) }[69,70]\end{array}$ \\
\hline $\begin{array}{l}\text { Executive function and } \\
\text { processing speed }\end{array}$ & $\begin{array}{l}\text { Trail Making Test A (established score } \\
\text { considering time, final number, and non- } \\
\text { corrected mistakes, a higher score indi- } \\
\text { cates better performance) }[71,72]\end{array}$ \\
\hline $\begin{array}{l}\text { Attention and working } \\
\text { memory }\end{array}$ & $\begin{array}{l}\text { Digit Span forward and backward } \\
\text { (number of correct digit spans) [73] }\end{array}$ \\
\hline
\end{tabular}

${ }^{\mathrm{a}}$ Standing balance, gait speed, and modified 5 times sit-to-stand (with using arms)

protocol analysis including participants who had a MEP adherence of at least 75\% (only in IG) and a complete assessment of spatiotemporal gait parameters in at least one condition of the gait assessment using GAITRite (i.e. single or two dual task conditions). Additionally, we implemented an intention-to-treat analysis and used multiple imputation (fully conditional specification imputation method, ten imputations, and ten iterations) to account for missing data. However, we did not impute data of deceased participants. To ensure plausibility of imputed data in the intention-to-treat analysis, we defined the following constraints: gait performance as both outcome and predictor variable, adherence to the MEP as well as related motor and cognitive performance as predictor variables only; minimum and maximum values according to observed range in each variable; rounding according to original data; and 100 maximal case draws, ten maximal parameter draws. We considered pooled results as provided by SPSS or reported ranges observed throughout the imputations, if SPSS did not support the pooling procedure, as final point estimates.

Required assumptions were tested before performing statistical analyses. For comparison of baseline values and sample characteristics between IG and CG, we used Chi-square tests, Mann-Whitney-U-Tests, and unpaired $\mathrm{T}$-Tests according to the scaling of the investigated outcome. We analyzed treatment effects using two-factor ANOVA with repeated measurements (time*group effects), and supplemented paired T-Tests (within group time effects). A two-sided $p$-value less than 0.05 
indicated statistical significance. To account for multiple comparisons, we also considered adjusted significance levels using Bonferroni-Holm correction in primary analyses. Additionally, we calculated $95 \%$ confidence intervals of differences between baseline and postintervention assessments and partial Eta ${ }^{2}$.

In secondary exploratory analyses, we included walking speed, stride length, and double support of the per protocol IG sample and determined differences in baseline performance (i.e. balance, mobility, strength and function of lower limbs, executive function, attention, and working memory) and selected sample characteristics (i.e. severity of cognitive impairments, etiology of dementia, and use of walking aids) between positive, non-, and negative responders using Chi-square tests, Kruskal-Wallis-Tests, and one-factor ANOVA. For post-hoc analyses, we used Dunn-Bonferroni-Tests and Tukey-Kramer post-hoc tests, respectively. $\mathrm{R}$ and partial $\mathrm{Eta}^{2}$ served as effect sizes. We defined positive responders as those participants, who improved their gait performance at least $10 \%$ during the 16week MEP, while negative responders showed a decline of at least $10 \%$ in gait performance, and non-responders were participants with less than $10 \%$ improvement or decline. This definition was based on percentage minimal detectable changes at $95 \%$ confidence interval of considered spatiotemporal gait parameters which ranged between 7 and $12 \%$ in a reliability study using GAITRite [58, 74]. The minimal detectable change is a measure of absolute reliability, which delineates "expected" from "true" changes in performance [57]. Moreover, we assessed the potential impact of changes in underlying motor and cognitive performance on changes in gait performance using multiple linear regression models with stepwise selection. Based on theoretical assumptions, we considered changes in balance, mobility, strength and function of lower limbs, executive function, attention, and working memory as independent variables. The calculated effect size is $\mathrm{f}^{2}$.

\section{Results}

\section{Recruitment and flow of participants}

Recruitment took place between March 2015 and March 2017. We screened 600 IWD for eligibility, of whom 319 were enrolled in the study. Of these, 201 participants were allocated to the IG and 118 to the CG. There was a dropout rate of $8 \%$ in both IG and CG, respectively. Reasons for dropouts are given in Fig. 2. There were no statistically significant differences in sample characteristics or baseline measurements between participants who dropped out versus those who completed the study. The mean adherence in the IG was $62 \%$. Overall, 107 participants (53\%) of the IG completed the MEP in accordance with the study protocol, i.e. defined by a minimum adherence of at least $75 \%$ of all sessions. $65 \%$ of participants in the IG and $62 \%$ of participants in the CG completed at least one condition of gait analysis at baseline and post-intervention assessment. Based on the above-mentioned criteria, 163 participants could be considered for the per protocol analysis. Even though we extended our initially planned recruitment phase for an additional year, we were not successful in reaching our intended sample size of 405 participants. This is due to the fact that the number of participants who did not fulfill our inclusion and exclusion criteria was much larger than expected. Nevertheless, a sensitivity analysis using G*Power 3 (Version 3.1.9.2 [55]) showed that we were still able to detect small to medium effects with our actual sample size $\left(\eta^{2}=0.013\right.$ to 0.018$)$. Figure 2 displays the flow of participants and states the reasons for dropouts and non- participations in assessments.

\section{Sample characteristics}

Table 2 provides an overview of the characteristics of participants at baseline (per protocol analysis; see Additional file 1 for sample characteristics of the intentionto-treat analysis). High rates of dependency on walking aids and a high mean CIRS morbidity index as well as number of required medications may indicate presence of medical comorbidities in the sample. We observed no statistically significant differences in characteristics between the IG and CG, except for the number of medications.

\section{Effects of the multimodal exercise program on spatiotemporal gait parameters \\ Per protocol analysis}

Participants of the IG (per protocol sample) had a mean adherence of $92 \%$. Table 3 presents baseline and postintervention values, differences between baseline and post-intervention assessments, group differences at baseline, within group time effects, and time*group effects including effect sizes of spatiotemporal gait parameters of the right leg for single and dual task conditions as well as dual task costs. We did not observe any statistically significant time"group effects. Furthermore, results on gait parameters of the left leg were comparable.

\section{Intention-to-treat analysis}

Missing data analysis showed an amount of missing data ranging between $8.5 \%$ (single task condition at baseline) and $47.6 \%$ (dual task counting backwards at postintervention assessment). With respect to gait performance, 194 of 319 records were incomplete. Reasons for missing values included not participating at postintervention assessment (see Fig. 2), weak physical condition, medical constrains, refusal, discontinuation of the assessment, invalid gait or dual task performance, and technical problems. Participants with incomplete data showed lower cognitive, motor, and gait performance, 


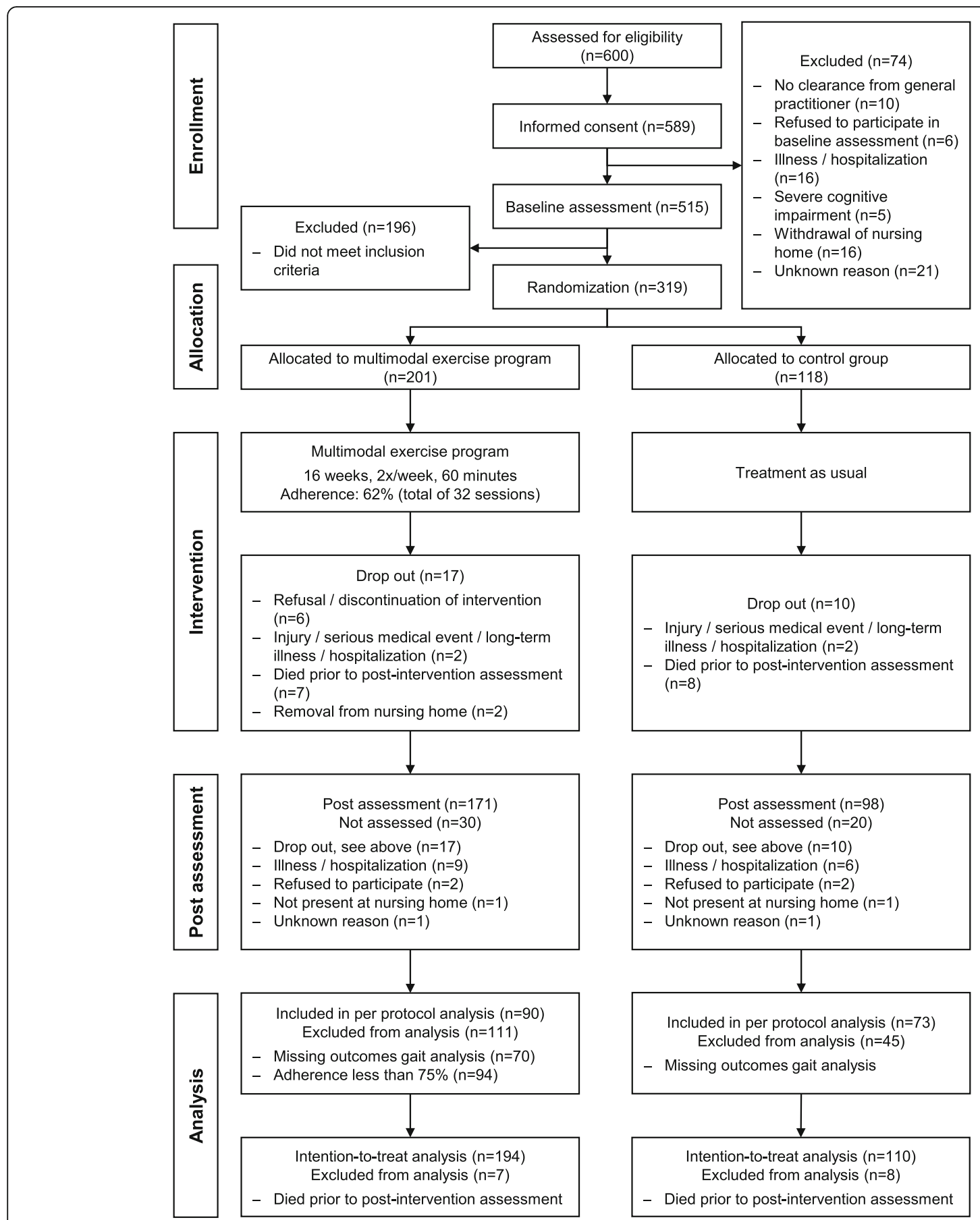

Fig. 2 Flow of participants (n: number)

were older, required more medication, and had worse CIRS scores, depending on walking condition and time of assessment. Accordingly, we assumed missing at random situation, which is necessary to apply multiple imputation.

Findings of the intention-to-treat analysis were comparable to those shown in the per protocol analysis, i.e. we did not observe any statistically significant time*group effects. Please refer to Additional file 2 for results of the intention-to-treat analysis.

Differences in characteristics between positive, negative, and non-responders (intervention group, per protocol analysis)

When taking into account walking speed, stride length, and double support in all three walking 
Table 2 Sample characteristics of participants at baseline (per protocol analysis)

\begin{tabular}{|c|c|c|c|}
\hline & $\begin{array}{l}\text { Intervention group } \\
{[n=90]}\end{array}$ & $\begin{array}{l}\text { Control group } \\
{[n=73]}\end{array}$ & $\begin{array}{l}\text { Group differences } \\
{\left[\mathrm{t}(\mathrm{df}) / / / \mathrm{Chi}^{2}(\mathrm{df}), \mathrm{p}\right]}\end{array}$ \\
\hline $\begin{array}{l}\text { Age, years } \\
{[\mathrm{M}(\mathrm{SD}) \text {, range] }}\end{array}$ & $85(7), 67-97$ & $86(5), 70-98$ & $\mathrm{t}(160.931)=1.918, p=0.057$ \\
\hline Sex, female & $82 \%$ & $86 \%$ & $\operatorname{Chi}^{2}(1)=0.500, p=0.479$ \\
\hline Type of dementia & & & $\mathrm{Chi}^{2}=5.693, p=0.199$ \\
\hline - Alzheimer's disease & $14 \%$ & $15 \%$ & \\
\hline - Vascular dementia & $21 \%$ & $11 \%$ & \\
\hline - Mixed dementia & $2 \%$ & $4 \%$ & \\
\hline - other & $2 \%$ & $0 \%$ & \\
\hline - unknown & $31 \%$ & $43 \%$ & \\
\hline - no confirmed/unknown diagnosis & $29 \%$ & $27 \%$ & \\
\hline MMSE [M (SD), range] & $17(4), 10-24$ & $17(4), 10-24$ & $\mathrm{t}(160.446)=0.317, p=0.752$ \\
\hline Use of walking aid & & & $\operatorname{Chi}^{2}(2)=4.644, p=0.098$ \\
\hline - walker & $62 \%$ & $77 \%$ & \\
\hline - walking stick/s & $9 \%$ & $8 \%$ & \\
\hline - no walking aid & $29 \%$ & $15 \%$ & \\
\hline \multicolumn{4}{|l|}{ CIRS [M (SD), range] } \\
\hline - Morbidity Index & $9(4), 1-20$ & $8(5), 2-26$ & $\mathrm{t}(101)=-0.633, p=0.528$ \\
\hline - Severity Index & $\begin{array}{l}1.5(0.4), 1-3 \\
\text { not available for } 31 \%\end{array}$ & $\begin{array}{l}1.5(0.4), 1-3 \\
\text { not available for } 44 \%\end{array}$ & $z=-0.247, p=0.805$ \\
\hline Number of medications $[\mathrm{M}(\mathrm{SD})$, range] & $\begin{array}{l}8(4), 1-27 \\
\text { unknown in 22\% }\end{array}$ & $\begin{array}{l}5(3), 0-12 \\
\text { unknown in 21\% }\end{array}$ & $\mathrm{t}(126)=-3.627, p<0.001$ \\
\hline $\begin{array}{l}\mathrm{BMI}, \mathrm{kg} / \mathrm{m}^{2} \\
{[\mathrm{M}(\mathrm{SD}), \text { range] }}\end{array}$ & $\begin{array}{l}28.4(4.4), 19.7-48.5 \\
\text { unknown in } 3 \%\end{array}$ & $\begin{array}{l}27.1(4.6), 17.6-36.5 \\
\text { unknown in } 3 \%\end{array}$ & $t(156)=-1.801, p=0.074$ \\
\hline
\end{tabular}

BMI: Body Mass Index, CIRS: Cumulative Illness Rating Scale, $d f$ : degree of freedom, M: mean, MMSE: Mini-Mental State Examination, $n$ : number, SD: standard deviation

Statistically significant results appear bold

conditions, between 10 and $39 \%$ of participants in the IG improved their gait performance by at least $10 \%$ (considered as positive responders). Moreover, 23 to $61 \%$ of IG participants did not change their gait performance (considered as non-responders), while 19 to $39 \%$ showed a decline in gait performance by at least $10 \%$ (considered as negative responders). Table 4 displays the proportion of positive, non-, and, negative responders in the IG depending on spatiotemporal gait parameter and walking condition, as well as mean changes in gait performance.

Positive, non-, and negative responders differed statistically significantly in terms of baseline performance of walking speed (both dual tasks), stride length (single task, dual task naming animals), double support (single task, both dual tasks), Timed Up \& Go Test (TUG; single task: stride length, dual task naming animals: walking speed), modified 30s CST (single task: double support), MMSE (single task), and proportion of walking aids (dual task naming animals: stride length; see Table 5, Additional file 3 presents statistically non-significant results).
The post-hoc analysis (see Table 5) revealed statistically significantly worse performance of positive compared to negative responders for walking speed (both dual tasks), stride length (single task, dual task naming animals), and double support (single task, both dual tasks). Besides we found worse performance of positive compared to non-responders for stride length (single task), double support (single task, dual task naming animals), TUG (single task: stride length), and modified 30s CST (single task: double support), as well as better performance of non- compared to negative responders for MMSE (single task).

\section{Impact of changes in underlying motor and cognitive performance on changes in gait performance (intervention group, per protocol analysis)}

Several weak to moderate correlations $(|r|=0.248$ $0.436, p<0.05)$ suggested relations of changes in underlying motor and cognitive performance with changes in gait performance in single and both dual task conditions. Multiple regression analyses revealed that changes in underlying motor and cognitive performance had an 
Table 3 Effects of the multimodal exercise program on spatiotemporal gait parameters and dual task costs (per protocol analysis)

\begin{tabular}{|c|c|c|c|c|c|c|c|c|}
\hline & & \multirow{2}{*}{$\begin{array}{l}\text { Baseline } \\
\text { [M (SD)] }\end{array}$} & \multirow{2}{*}{$\begin{array}{l}\text { Group differences } \\
\text { at baseline } \\
\text { [t (df), p] }\end{array}$} & \multirow{2}{*}{$\begin{array}{l}\text { Post } \\
{[\mathrm{M}(\mathrm{SD})]}\end{array}$} & \multirow{2}{*}{$\begin{array}{l}\text { Difference post - } \\
\text { baseline } \\
{\left[\mathrm{M}(\mathrm{SD}),\left[\mathrm{Cl}_{95}\right]\right]}\end{array}$} & \multirow{2}{*}{$\begin{array}{l}\text { Within group } \\
\text { time effects } \\
{[t(d f), p]}\end{array}$} & \multicolumn{2}{|c|}{ Time* group effects } \\
\hline & & & & & & & $\begin{array}{l}\mathrm{F}\left(\mathrm{df}_{\text {numerator, }}\right. \\
\mathrm{df} \text { denominator }), \mathrm{p}\end{array}$ & $\begin{array}{l}\text { Effect size } \\
\eta_{p}^{2}\end{array}$ \\
\hline \multicolumn{9}{|c|}{ Single task (IG: $n=89, C G: n=73$ ) } \\
\hline \multirow[t]{2}{*}{ Walking speed, $\mathrm{m} / \mathrm{sec}$} & IG & $\begin{array}{l}0.67 \\
(0.19)\end{array}$ & $\begin{array}{l}\mathrm{t}(160)=-1.659 \\
p=0.099\end{array}$ & $\begin{array}{l}0.65 \\
(0.22)\end{array}$ & $\begin{array}{l}-0.02(0.13) \\
{[-0.05,0.00]}\end{array}$ & $\begin{array}{l}\mathrm{t}(88)=1.787 \\
p=0.077\end{array}$ & $\begin{array}{l}F(1,160)=0.036 \\
p=0.849\end{array}$ & 0.000 \\
\hline & CG & $\begin{array}{l}0.62 \\
(0.19)\end{array}$ & & $\begin{array}{l}0.60 \\
(0.20)\end{array}$ & $\begin{array}{l}-0.02(0.13) \\
{[-0.05,0.01]}\end{array}$ & $\begin{array}{l}t(72)=1.373 \\
p=0.174\end{array}$ & & \\
\hline \multirow[t]{2}{*}{ Stride length, $\mathrm{cm}$} & IG & $\begin{array}{l}82.6 \\
(19.7)\end{array}$ & $\begin{array}{l}t(159.875)=-0.842 \\
p=0.401\end{array}$ & $\begin{array}{l}80.5 \\
(21.2)\end{array}$ & $\begin{array}{l}-2.1(10.9),[-4.4 \\
0.2]\end{array}$ & $\begin{array}{l}\mathrm{t}(88)=1.825 \\
p=0.071\end{array}$ & $\begin{array}{l}F(1,160)=0.030 \\
p=0.863^{a}\end{array}$ & 0.000 \\
\hline & CG & $\begin{array}{l}80.2 \\
(15.7)\end{array}$ & & $\begin{array}{l}77.8 \\
(16.9)\end{array}$ & $\begin{array}{l}-2.4(10.4),[-4.8, \\
0.0]\end{array}$ & $\begin{array}{l}\mathrm{t}(72)=1.973 \\
p=0.052\end{array}$ & & \\
\hline \multirow[t]{2}{*}{ Stride time, sec } & IG & $1.3(0.2)$ & $\begin{array}{l}t(131.361)=2.346 \\
p=0.020\end{array}$ & $1.3(0.2)$ & $0.0(0.2),[0.0,0.1]$ & $\begin{array}{l}\mathrm{t}(88)=-1.571 \\
p=0.120\end{array}$ & $\begin{array}{l}F(1,160)=0.195 \\
p=0.660^{a}\end{array}$ & 0.001 \\
\hline & CG & $1.3(0.2)$ & & $1.4(0.3)$ & $0.0(0.2),[0.0,0.1]$ & $\begin{array}{l}t(72)=-0.853 \\
p=0.397\end{array}$ & & \\
\hline \multirow[t]{2}{*}{$\begin{array}{l}\text { Double support, } \\
\% \text { of stride time }\end{array}$} & IG & $\begin{array}{l}38.0 \\
(8.1)\end{array}$ & $\begin{array}{l}t(160)=1.289 \\
p=0.199\end{array}$ & $\begin{array}{l}39.2 \\
(8.4)\end{array}$ & $1.1(4.9),[0.1,2.2]$ & $\begin{array}{l}\mathrm{t}(88)=-2.182 \\
p=0.032\end{array}$ & $\begin{array}{l}F(1,160)=0.005 \\
p=0.943\end{array}$ & 0.000 \\
\hline & CG & $\begin{array}{l}39.6 \\
(7.4)\end{array}$ & & $\begin{array}{l}40.8 \\
(7.3)\end{array}$ & $1.2(4.9),[0.0,2.3]$ & $\begin{array}{l}\mathrm{t}(72)=-2.070 \\
p=0.042\end{array}$ & & \\
\hline \multirow[t]{2}{*}{$\begin{array}{l}\text { Stance phase, } \\
\% \text { of stride time }\end{array}$} & $\mathrm{IG}$ & $\begin{array}{l}68.9 \\
(4.1)\end{array}$ & $\begin{array}{l}\mathrm{t}(160)=1.368 \\
p=0.173\end{array}$ & $\begin{array}{l}69.5 \\
(4.4)\end{array}$ & $0.6(2.5),[0.1,1.1]$ & $\begin{array}{l}\mathrm{t}(88)=-2.208 \\
p=0.030\end{array}$ & $\begin{array}{l}F(1,160)=0.004 \\
p=0.949\end{array}$ & 0.000 \\
\hline & CG & $\begin{array}{l}69.8 \\
(4.1)\end{array}$ & & $\begin{array}{l}70.4 \\
(4.2)\end{array}$ & $0.6(3.1),[-0.2,1.3]$ & $\begin{array}{l}\mathrm{t}(72)=-1.543 \\
p=0.127\end{array}$ & & \\
\hline \multicolumn{9}{|c|}{ Dual task, counting backwards (IG: $n=62, C G: n=52$ ) } \\
\hline \multirow[t]{2}{*}{$\begin{array}{l}\text { Walking speed, } \\
\mathrm{m} / \mathrm{sec}\end{array}$} & IG & $\begin{array}{l}0.55 \\
(0.16)\end{array}$ & $\begin{array}{l}t(112)=-2.236, p= \\
0.027\end{array}$ & $\begin{array}{l}0.54 \\
(0.16)\end{array}$ & $\begin{array}{l}-0.02(0.14),[- \\
0.06,0.02]\end{array}$ & $\begin{array}{l}\mathrm{t}(61)=1.001 \\
p=0.321\end{array}$ & $\begin{array}{l}F(1,112)=0.101 \\
p=0.752\end{array}$ & 0.001 \\
\hline & CG & $\begin{array}{l}0.48 \\
(0.17)\end{array}$ & & $\begin{array}{l}0.47 \\
(0.16)\end{array}$ & $\begin{array}{l}-0.01(0.15),[- \\
0.05,0.03]\end{array}$ & $\begin{array}{l}t(51)=0.470 \\
p=0.641\end{array}$ & & \\
\hline \multirow[t]{2}{*}{$\begin{array}{l}\text { Stride length, } \\
\mathrm{cm}\end{array}$} & IG & $\begin{array}{l}78.2 \\
(19.1)\end{array}$ & $\begin{array}{l}\mathrm{t}(112)=-1.407 \\
p=0.162\end{array}$ & $\begin{array}{l}78.8 \\
(19.5)\end{array}$ & $\begin{array}{l}0.5(11.6),[-2.4, \\
3.5]\end{array}$ & $\begin{array}{l}t(61)=-0.359 \\
p=0.721\end{array}$ & $\begin{array}{l}F(1,112)=0.193 \\
p=0.661\end{array}$ & 0.002 \\
\hline & CG & $\begin{array}{l}73.5 \\
(16.6)\end{array}$ & & $\begin{array}{l}75.1 \\
(16.6)\end{array}$ & $\begin{array}{l}1.6(15.3),[-2.6, \\
5.9]\end{array}$ & $\begin{array}{l}\mathrm{t}(51)=-0.773 \\
p=0.443\end{array}$ & & \\
\hline \multirow[t]{2}{*}{$\begin{array}{l}\text { Stride time, } \\
\mathrm{sec}\end{array}$} & $\mathrm{IG}$ & $1.5(0.3)$ & $\begin{array}{l}t(95.044)=2.446 \\
p=0.016\end{array}$ & $1.5(0.4)$ & $0.1(0.4),[0.0,0.2]$ & $\begin{array}{l}\mathrm{t}(61)=-1.605 \\
p=0.114\end{array}$ & $\begin{array}{l}F(1,112)=0.253 \\
p=0.616^{a, b}\end{array}$ & 0.002 \\
\hline & CG & $1.6(0.3)$ & & $1.7(0.5)$ & $0.1(0.4),[0.0,0.2]$ & $\begin{array}{l}t(51)=-2.149 \\
p=0.036\end{array}$ & & \\
\hline \multirow[t]{2}{*}{$\begin{array}{l}\text { Double support, } \\
\% \text { of stride time }\end{array}$} & $\mathrm{IG}$ & $\begin{array}{l}40.6 \\
(9.3)\end{array}$ & $\begin{array}{l}t(112)=2.110 \\
p=0.037\end{array}$ & $\begin{array}{l}41.3 \\
(8.7)\end{array}$ & $0.7(5.9),[-0.8,2.2]$ & $\begin{array}{l}\mathrm{t}(61)=-0.998 \\
p=0.322\end{array}$ & $\begin{array}{l}F(1,112)=0.042 \\
p=0.839\end{array}$ & 0.000 \\
\hline & CG & $\begin{array}{l}44.2 \\
(9.1)\end{array}$ & & $\begin{array}{l}44.7 \\
(9.0)\end{array}$ & $0.5(7.4),[-1.6,2.6]$ & $\begin{array}{l}t(51)=-0.481 \\
p=0.632\end{array}$ & & \\
\hline \multirow[t]{2}{*}{$\begin{array}{l}\text { Stance phase, } \\
\% \text { of stride time }\end{array}$} & IG & $\begin{array}{l}70.3 \\
(5.2)\end{array}$ & $\begin{array}{l}t(112)=1.850 \\
p=0.067\end{array}$ & $\begin{array}{l}70.4 \\
(4.7)\end{array}$ & $0.2(2.9),[-0.6,0.9]$ & $\begin{array}{l}t(61)=-0.414 \\
p=0.680\end{array}$ & $\begin{array}{l}F(1,112)=0.009 \\
p=0.925\end{array}$ & 0.000 \\
\hline & CG & $\begin{array}{l}72.1 \\
(5.2)\end{array}$ & & $\begin{array}{l}72.3 \\
(5.2)\end{array}$ & $0.2(4.3),[-1.0,1.4]$ & $\begin{array}{l}t(51)=-0.359 \\
p=0.721\end{array}$ & & \\
\hline \multicolumn{9}{|c|}{ Dual-task costs, counting backwards (IG: $n=62, C G: n=52)$} \\
\hline \multirow[t]{2}{*}{$\begin{array}{l}\text { Walking speed, } \\
\%\end{array}$} & IG & $\begin{array}{l}-20.5 \\
(15.2)\end{array}$ & $\begin{array}{l}t(112)=-1.105 \\
p=0.271\end{array}$ & $\begin{array}{l}-21.0 \\
(15.5)\end{array}$ & $\begin{array}{l}-0.6(16.6),[-4.8, \\
3.6]\end{array}$ & $\begin{array}{l}t(61)=0.278 \\
p=0.782\end{array}$ & $\begin{array}{l}F(1,112)=0.053 \\
p=0.818\end{array}$ & 0.000 \\
\hline & CG & $\begin{array}{l}-23.5 \\
(14.0)\end{array}$ & & $\begin{array}{l}-23.4 \\
(17.3)\end{array}$ & $\begin{array}{l}0.1(17.3),[-4.7, \\
5.0]\end{array}$ & $\begin{array}{l}\mathrm{t}(51)=-0.061 \\
p=0.952\end{array}$ & & \\
\hline \multirow[t]{2}{*}{$\begin{array}{l}\text { Stride length, } \\
\%\end{array}$} & $\mathrm{IG}$ & $\begin{array}{l}-8.8 \\
(11.7)\end{array}$ & $\begin{array}{l}t(112)=-0.853 \\
p=0.395\end{array}$ & $\begin{array}{l}-7.0 \\
(9.4)\end{array}$ & $\begin{array}{l}1.7(13.0),[-1.6, \\
5.0]\end{array}$ & $\begin{array}{l}t(61)=-1.042 \\
p=0.302\end{array}$ & $\begin{array}{l}F(1,112)=0.759 \\
p=0.386^{b}\end{array}$ & 0.007 \\
\hline & CG & $\begin{array}{l}-10.5 \\
(9.9)\end{array}$ & & $\begin{array}{l}-6.6 \\
(14.6)\end{array}$ & $3.9(13.6),[0.1,7.7]$ & $\begin{array}{l}t(51)=-2.064 \\
p=0.044\end{array}$ & & \\
\hline
\end{tabular}


Table 3 Effects of the multimodal exercise program on spatiotemporal gait parameters and dual task costs (per protocol analysis) (Continued)

\begin{tabular}{|c|c|c|c|c|c|c|c|c|}
\hline & & \multirow{2}{*}{$\begin{array}{l}\text { Baseline } \\
\text { [M (SD)] }\end{array}$} & \multirow{2}{*}{$\begin{array}{l}\text { Group differences } \\
\text { at baseline } \\
{[t(d f), p]}\end{array}$} & \multirow{2}{*}{$\begin{array}{l}\text { Post } \\
{[M(S D)]}\end{array}$} & \multirow{2}{*}{$\begin{array}{l}\text { Difference post - } \\
\text { baseline } \\
{\left[\mathrm{M}(\mathrm{SD}),\left[\mathrm{Cl}_{95}\right]\right]}\end{array}$} & \multirow{2}{*}{$\begin{array}{l}\text { Within group } \\
\text { time effects } \\
{[t(d f), p]}\end{array}$} & \multicolumn{2}{|c|}{ Time* group effects } \\
\hline & & & & & & & $\begin{array}{l}\mathrm{F}\left(\mathrm{df}_{\text {numerator }}\right. \\
\left.\mathrm{df}_{\text {denominator }}\right), \mathrm{p}\end{array}$ & $\begin{array}{l}\text { Effect size } \\
\eta_{p}^{2}\end{array}$ \\
\hline \multirow[t]{2}{*}{ Stride time, $\%$} & IG & $\begin{array}{l}17.3 \\
(17.3)\end{array}$ & $\begin{array}{l}\mathrm{t}(112)=0.806 \\
p=0.422\end{array}$ & $\begin{array}{l}21.0 \\
(23.9)\end{array}$ & $\begin{array}{l}3.7(25.7),[-2.8, \\
10.2]\end{array}$ & $\begin{array}{l}\mathrm{t}(61)=-1.130 \\
p=0.263\end{array}$ & $\begin{array}{l}F(1,112)=0.257 \\
p=0.613\end{array}$ & 0.002 \\
\hline & CG & $\begin{array}{l}19.9 \\
(17.1)\end{array}$ & & $\begin{array}{l}25.9 \\
(24.3)\end{array}$ & $\begin{array}{l}6.0(22.8),[-0.3, \\
12.4]\end{array}$ & $\begin{array}{l}t(51)=-1.905 \\
p=0.062\end{array}$ & & \\
\hline \multirow[t]{2}{*}{ Double support, $\%$} & IG & $\begin{array}{l}11.0 \\
(14.3)\end{array}$ & $\begin{array}{l}\mathrm{t}(112)=0.305 \\
p=0.761\end{array}$ & $\begin{array}{l}11.9 \\
(12.8)\end{array}$ & $\begin{array}{l}1.0(15.5),[-3.0, \\
4.9]\end{array}$ & $\begin{array}{l}t(61)=-0.491 \\
p=0.625\end{array}$ & $\begin{array}{l}F(1,112)=0.081 \\
p=0.776\end{array}$ & 0.001 \\
\hline & CG & $\begin{array}{l}11.7 \\
(10.8)\end{array}$ & & $\begin{array}{l}11.8 \\
(14.8)\end{array}$ & $\begin{array}{l}0.1(15.1),[-4.1, \\
4.3]\end{array}$ & $\begin{array}{l}\mathrm{t}(51)=-0.069 \\
p=0.945\end{array}$ & & \\
\hline \multirow[t]{2}{*}{ Stance phase, $\%$} & IG & $3.1(4.5)$ & $\begin{array}{l}\mathrm{t}(112)=0.095 \\
p=0.924\end{array}$ & $2.9(3.6)$ & $\begin{array}{l}-0.2(4.8),[-1.4, \\
1.1]\end{array}$ & $\begin{array}{l}t(61)=0.252 \\
p=0.802\end{array}$ & $\begin{array}{l}F(1,112)=0.130 \\
p=0.719\end{array}$ & 0.001 \\
\hline & CG & $3.1(3.7)$ & & $3.3(4.8)$ & $0.2(5.5),[-1.3,1.7]$ & $\begin{array}{l}t(51)=-0.254 \\
p=0.800\end{array}$ & & \\
\hline \multicolumn{9}{|c|}{ Dual task, naming animals (IG: $n=61, C G: n=59$ ) } \\
\hline \multirow[t]{2}{*}{ Walking speed, $\mathrm{m} / \mathrm{sec}$} & IG & $\begin{array}{l}0.45 \\
(0.14)\end{array}$ & $\begin{array}{l}\mathrm{t}(118)=-1.797 \\
p=0.075\end{array}$ & $\begin{array}{l}0.43 \\
(0.13)\end{array}$ & $\begin{array}{l}-0.01(0.12),[- \\
0.04,0.02]\end{array}$ & $\begin{array}{l}t(60)=0.805 \\
p=0.424\end{array}$ & $\begin{array}{l}F(1,118)=0.972 \\
p=0.326\end{array}$ & 0.008 \\
\hline & CG & $\begin{array}{l}0.40 \\
(0.14)\end{array}$ & & $\begin{array}{l}0.41 \\
(0.13)\end{array}$ & $\begin{array}{l}0.01(0.12),[-0.02 \\
0.04]\end{array}$ & $\begin{array}{l}t(58)=-0.593 \\
p=0.555\end{array}$ & & \\
\hline \multirow[t]{2}{*}{ Stride length, $\mathrm{cm}$} & IG & $\begin{array}{l}70.4 \\
(18.1)\end{array}$ & $\begin{array}{l}\mathrm{t}(118)=-1.415 \\
p=0.160\end{array}$ & $\begin{array}{l}71.2 \\
(17.7)\end{array}$ & $\begin{array}{l}0.9(11.0),[-2.0, \\
3.7]\end{array}$ & $\begin{array}{l}t(60)=-0.620 \\
p=0.538\end{array}$ & $\begin{array}{l}F(1,118)=0.040 \\
p=0.841\end{array}$ & 0.000 \\
\hline & CG & $\begin{array}{l}65.9 \\
(16.3)\end{array}$ & & $\begin{array}{l}66.3 \\
(14.9)\end{array}$ & $\begin{array}{l}0.4(13.2),[-3.0, \\
3.9]\end{array}$ & $\begin{array}{l}t(58)=-0.252 \\
p=0.802\end{array}$ & & \\
\hline \multirow[t]{2}{*}{ Stride time, sec } & IG & $1.6(0.4)$ & $\begin{array}{l}\mathrm{t}(118)=1.480 \\
p=0.141\end{array}$ & $1.7(0.4)$ & $0.1(0.3),[0.0,0.2]$ & $\begin{array}{l}t(60)=-1.823 \\
p=0.073\end{array}$ & $\begin{array}{l}F(1,118)=3.448 \\
p=0.066\end{array}$ & 0.028 \\
\hline & CG & $1.7(0.5)$ & & $1.7(0.5)$ & $0.0(0.3),[-0.1,0.1]$ & $\begin{array}{l}t(58)=0.801 \\
p=0.426\end{array}$ & & \\
\hline \multirow[t]{2}{*}{$\begin{array}{l}\text { Double support, \% of } \\
\text { stride time }\end{array}$} & IG & $\begin{array}{l}45.9 \\
(9.4)\end{array}$ & $\begin{array}{l}\mathrm{t}(118)=1.526 \\
p=0.130\end{array}$ & $\begin{array}{l}45.2 \\
(8.6)\end{array}$ & $\begin{array}{l}-0.7(7.1),[-2.5 \\
1.1]\end{array}$ & $\begin{array}{l}\mathrm{t}(60)=0.758 \\
p=0.452\end{array}$ & $\begin{array}{l}F(1,118)=0.085 \\
p=0.771\end{array}$ & 0.001 \\
\hline & CG & $\begin{array}{l}48.5 \\
(9.5)\end{array}$ & & $\begin{array}{l}48.2 \\
(8.6)\end{array}$ & $\begin{array}{l}-0.3(7.2),[-2.2, \\
1.6]\end{array}$ & $\begin{array}{l}t(58)=0.326 \\
p=0.746\end{array}$ & & \\
\hline \multirow[t]{2}{*}{$\begin{array}{l}\text { Stance phase, \% of } \\
\text { stride time }\end{array}$} & IG & $\begin{array}{l}72.4 \\
(4.7)\end{array}$ & $\begin{array}{l}t(118)=2.233 \\
p=0.027\end{array}$ & $\begin{array}{l}72.3 \\
(4.4)\end{array}$ & $\begin{array}{l}-0.1(3.9),[-1.1, \\
0.9]\end{array}$ & $\begin{array}{l}\mathrm{t}(60)=0.241 \\
p=0.810\end{array}$ & $\begin{array}{l}F(1,118)=0.107 \\
p=0.744\end{array}$ & 0.001 \\
\hline & CG & $\begin{array}{l}74.5 \\
(5.5)\end{array}$ & & $\begin{array}{l}74.1 \\
(5.1)\end{array}$ & $\begin{array}{l}-0.3(3.7),[-1.3, \\
0.6]\end{array}$ & $\begin{array}{l}t(58)=0.727 \\
p=0.470\end{array}$ & & \\
\hline \multicolumn{9}{|c|}{ Dual-task costs, naming animals (IG: $n=60, C G: n=59$ ) } \\
\hline \multirow[t]{2}{*}{ Walking speed, $\%$} & $\mathrm{IG}$ & $\begin{array}{l}-34.4 \\
(15.9)\end{array}$ & $\begin{array}{l}\mathrm{t}(117)=-0.520 \\
p=0.604\end{array}$ & $\begin{array}{l}-32.4 \\
(18.6)\end{array}$ & $\begin{array}{l}2.0(19.7),[-3.1 \\
7.1]\end{array}$ & $\begin{array}{l}\mathrm{t}(59)=-0.776 \\
p=0.441\end{array}$ & $\begin{array}{l}F(1,117)=0.696 \\
p=0.406^{a}\end{array}$ & 0.006 \\
\hline & CG & $\begin{array}{l}-35.9 \\
(16.0)\end{array}$ & & $\begin{array}{l}-31.2 \\
(14.6)\end{array}$ & 4.8 (16.6), [0.4, 9.1] & $\begin{array}{l}t(58)=-2.204 \\
p=0.032\end{array}$ & & \\
\hline \multirow[t]{2}{*}{ Stride length, \% } & IG & $\begin{array}{l}-17.9 \\
(11.1)\end{array}$ & $\begin{array}{l}\mathrm{t}(117)=-0.406 \\
p=0.685\end{array}$ & $\begin{array}{l}-14.3 \\
(12.4)\end{array}$ & $\begin{array}{l}3.7 \text { (15.4), [- } 0.3 \text {, } \\
7.6]\end{array}$ & $\begin{array}{l}\mathrm{t}(59)=-1.842 \\
p=0.070\end{array}$ & $\begin{array}{l}F(1,117)=0.007 \\
p=0.931\end{array}$ & 0.000 \\
\hline & CG & $\begin{array}{l}-18.8 \\
(12.5)\end{array}$ & & $\begin{array}{l}-14.9 \\
(12.7)\end{array}$ & $3.9(12.9),[0.5,7.3]$ & $\begin{array}{l}t(58)=-2.316 \\
p=0.024\end{array}$ & & \\
\hline \multirow[t]{2}{*}{ Stride time, \% } & IG & $\begin{array}{l}30.1 \\
(27.1)\end{array}$ & $\begin{array}{l}\mathrm{t}(117)=0.326 \\
p=0.745\end{array}$ & $\begin{array}{l}33.2 \\
(29.8)\end{array}$ & $\begin{array}{l}3.2(30.3),[-4.7, \\
11.0]\end{array}$ & $\begin{array}{l}t(59)=-0.807 \\
p=0.423\end{array}$ & $\begin{array}{l}F(1,117)=2.558 \\
p=0.112^{b}\end{array}$ & 0.021 \\
\hline & CG & $\begin{array}{l}31.6 \\
(24.6)\end{array}$ & & $\begin{array}{l}27.2 \\
(22.7)\end{array}$ & $\begin{array}{l}-4.4(20.4),[-9.7, \\
0.9]\end{array}$ & $\begin{array}{l}t(58)=1.669 \\
p=0.100\end{array}$ & & \\
\hline \multirow[t]{2}{*}{ Double support, \% } & IG & $\begin{array}{l}25.4 \\
(17.6)\end{array}$ & $\begin{array}{l}\mathrm{t}(117)=-0.643 \\
p=0.522\end{array}$ & $\begin{array}{l}21.5 \\
(17.8)\end{array}$ & $\begin{array}{l}-3.9(21.7),[-9.5 \\
1.7]\end{array}$ & $\begin{array}{l}t(59)=1.401 \\
p=0.166\end{array}$ & $\begin{array}{l}F(1,117)=0.005 \\
p=0.946\end{array}$ & 0.000 \\
\hline & CG & $\begin{array}{l}23.4 \\
(15.6)\end{array}$ & & $\begin{array}{l}19.3 \\
(16.2)\end{array}$ & $\begin{array}{l}-4.2(15.7),[-8.3 \\
-0.1]\end{array}$ & $\begin{array}{l}\mathrm{t}(58)=2.027 \\
p=0.047\end{array}$ & & \\
\hline
\end{tabular}


Table 3 Effects of the multimodal exercise program on spatiotemporal gait parameters and dual task costs (per protocol analysis) (Continued)

\begin{tabular}{|c|c|c|c|c|c|c|c|c|}
\hline & & \multirow{2}{*}{$\begin{array}{l}\text { Baseline } \\
\text { [M (SD)] }\end{array}$} & \multirow{2}{*}{$\begin{array}{l}\text { Group differences } \\
\text { at baseline } \\
{[t(d f), p]}\end{array}$} & \multirow{2}{*}{$\begin{array}{l}\text { Post } \\
{[M(S D)]}\end{array}$} & \multirow{2}{*}{$\begin{array}{l}\text { Difference post - } \\
\text { baseline } \\
{\left[\mathrm{M}(\mathrm{SD}),\left[\mathrm{Cl}_{95}\right]\right]}\end{array}$} & \multirow{2}{*}{$\begin{array}{l}\text { Within group } \\
\text { time effects } \\
{[t(d f), p]}\end{array}$} & \multicolumn{2}{|c|}{ Time* group effects } \\
\hline & & & & & & & $\begin{array}{l}\mathrm{F}\left(\mathrm{df} f_{\text {numeratorr }}\right. \\
\left.\mathrm{df} f_{\text {denominator }}\right), p\end{array}$ & $\begin{array}{l}\text { Effect size } \\
\eta_{p}{ }^{2}\end{array}$ \\
\hline \multirow[t]{2}{*}{ Stance phase, $\%$} & IG & $6.3(4.5)$ & $\begin{array}{l}t(117)=0.832 \\
p=0.407\end{array}$ & $5.5(5.0)$ & $\begin{array}{l}-0.7(6.0) \\
{[-2.3,0.8]}\end{array}$ & $\begin{array}{l}\mathrm{t}(59)=0.986 \\
p=0.337\end{array}$ & $\begin{array}{l}F(1,117)=0.389 \\
p=0.534\end{array}$ & 0.003 \\
\hline & CG & $7.0(4.9)$ & & $5.6(4.6)$ & $\begin{array}{l}-1.4(4.8) \\
{[-2.6,-0.1]}\end{array}$ & $\begin{array}{l}\mathrm{t}(58)=2.183 \\
p=0.033\end{array}$ & & \\
\hline
\end{tabular}

Statistically significant results appear bold for $a=0.05$. When considering adjusted significance levels using Bonferroni-Holm correction for multiple comparisons, no statistically significant results were observed

CG: control group, $C l_{95}$ : 95\% confidence interval, $d f$ degrees of freedom, IG: intervention group, $M:$ mean, $n$ : number, SD: standard deviation

avariance homogeneity not fulfilled

${ }^{\mathrm{b}}$ covariance homogeneity not fulfilled

impact on changes in gait performance. Related models were statistically significant and explained 12.6 to $39.4 \%$ of the overall variance. Changes in TUG, modified 30s CST, modified Short Physical Performance Battery (SPPB), Clock Drawing Test, Digit Span forward and backward, and Trail Making Test had statistically significant regression coefficients $(p<0.05)$. Generally, improvements in gait performance were associated with improvements in motor and cognitive assessments. Table 6 presents the details of the multiple regression analysis models.

\section{Discussion}

\section{Effects of the multimodal exercise program on} spatiotemporal gait parameters

This multicenter randomized controlled trial aimed to investigate the effectiveness of a dementia-specific MEP, which combined motor and cognitive tasks, on gait performance. As we did not observe any statistically significant time"group effects, our primary hypothesis that a 16-week MEP may have a differential effect on gait performance in IWD as compared to conventional treatment alone could not be confirmed. This may be explained by the heterogeneity of the study sample or the relatively low amount of walking tasks included in the intervention.

With regard to sample characteristics as well as motor, cognitive, and gait performance at baseline, we observed large standard deviations indicating that the sample of IWD was highly heterogeneous in our study (see Tables 2 and 3, and Additional file 4). Due to this large heterogeneity, it was very difficult to adequately tailor one standardized physical activity intervention to the needs of all participants, and we postulate that there is likely no standard physical activity intervention that fits all IWD.

With respect to the applied intervention, an analysis of components of the MEP showed that it did not include a large amount of specific walking tasks. Even though we had planned to increase the number of exercises focusing on walking throughout the intervention, this was

Table 4 Positive, non-, and negative responders in the intervention group and mean changes in gait performance (per protocol analysis)

\begin{tabular}{|c|c|c|c|}
\hline All & Negative responders & Non-responders & Positive responders \\
\hline Mean change (SD) & Mean change (SD) & Mean change (SD) & Mean change (SD) \\
\hline
\end{tabular}

\begin{tabular}{|c|c|c|c|c|c|c|c|c|}
\hline \multicolumn{9}{|l|}{ Single task } \\
\hline Walking speed, $\mathrm{m} / \mathrm{sec}$ & 89 & $-0.03(0.21)$ & $35 \%$ & $-0.22(0.09)$ & $48 \%$ & $-0.01(0.05)$ & $17 \%$ & $0.32(0.19)$ \\
\hline Stride length, $\mathrm{cm}$ & 89 & $-2.07(14.98)$ & $26 \%$ & $-19.59(10.15)$ & $57 \%$ & $-0.75(5.42)$ & $17 \%$ & $20.30(9.81)$ \\
\hline Double support, $\%$ of stride time & 89 & $3.57(12.58)$ & $29 \%$ & $18.99(8.62)$ & $61 \%$ & $-0.57(4.69)$ & $10 \%$ & $-16.15(6.08)$ \\
\hline \multicolumn{9}{|l|}{ Dual task, counting backwards } \\
\hline Walking speed, $\mathrm{m} / \mathrm{sec}$ & 62 & $0(0.26)$ & $39 \%$ & $-0.26(0.11)$ & $23 \%$ & $-0.02(0.04)$ & $39 \%$ & $0.27(0.14)$ \\
\hline Stride length, $\mathrm{cm}$ & 62 & $2.06(17.08)$ & $19 \%$ & $-18.36(7.05)$ & $50 \%$ & $-2.18(4.88)$ & $31 \%$ & $21.87(13.99)$ \\
\hline Double support, $\%$ of stride time & 62 & $3.16(14.32)$ & $29 \%$ & $20.67(9.37)$ & $55 \%$ & $-0.36(5.56)$ & $16 \%$ & $-16.44(4.76)$ \\
\hline \multicolumn{9}{|l|}{ Dual task, naming animals } \\
\hline Walking speed, $\mathrm{m} / \mathrm{sec}$ & 61 & $0.02(0.29)$ & $34 \%$ & $-0.27(0.12)$ & $31 \%$ & $-0.01(0.06)$ & $34 \%$ & $0.33(0.23)$ \\
\hline Stride length, $\mathrm{cm}$ & 61 & $3.49(19.36)$ & $23 \%$ & $-18.66(7.34)$ & $49 \%$ & $0.94(6.27)$ & $28 \%$ & $26.21(17.33)$ \\
\hline Double support, $\%$ of stride time & 61 & $-0.14(14.68)$ & $23 \%$ & $21.12(9.04)$ & $56 \%$ & $-1.99(4.65)$ & $21 \%$ & $-18.20(6.27)$ \\
\hline
\end{tabular}

$n$ : number, SD: standard deviation 
Table 5 Statistically significant differences in baseline motor and cognitive performance as well as the use of walking aids between positive, non-, and negative responders in the intervention group (per protocol analysis)

\begin{tabular}{|c|c|c|c|c|c|}
\hline & $\begin{array}{l}\text { Negative } \\
\text { responders }\end{array}$ & $\begin{array}{l}\text { Non- } \\
\text { responders }\end{array}$ & $\begin{array}{l}\text { Positive } \\
\text { responders }\end{array}$ & Between group difference & Post-hoc analysis \\
\hline & Mean (SD) & Mean (SD) & Mean (SD) & $\begin{array}{l}\mathrm{F}\left(\mathrm{df}_{\text {numerator, }} \mathrm{df}_{\text {denominator }}\right) / \\
\mathrm{Chi}^{2}(\mathrm{df}), \mathrm{p}\end{array}$ & \\
\hline \multicolumn{6}{|l|}{ Single task, walking speed } \\
\hline MMSE $(n=89)$ & $14.8(4.0)$ & $18.5(3.9)$ & $16.7(5.1)$ & $\mathrm{Chi}^{2}(2)=12.093, p=0.002$ & $z=-3.472, p=0.002, r=0.404^{a}$ \\
\hline \multicolumn{6}{|l|}{ Single task, stride length } \\
\hline Stride length, $\mathrm{cm}(\mathrm{n}=89)$ & $80.3(19.9)$ & $89.4(15.2)$ & $62.7(20.0)$ & $\begin{array}{l}F(2,86)=14.129, p<0.001 \\
\eta_{p}^{2}=0.247\end{array}$ & $\begin{array}{l}p=0.008, \mathrm{MD}=-17.60, \mathrm{Cl}_{95}[-31.32, \\
-3.89]^{\mathrm{b}} \\
\mathrm{p}<0.001, \mathrm{MD}=-26.79, \mathrm{Cl}_{95}[- \\
38.93,-14.65]^{c}\end{array}$ \\
\hline TUG, sec $(n=89)$ & $22.9(10.9)$ & $19.0(7.6)$ & $31.5(20.0)$ & $\mathrm{Chi}^{2}(2)=8.234, \mathrm{p}=0.016$ & $z=-2.800, p=0.015, r=0.325^{c}$ \\
\hline MMSE $(n=89)$ & $14.5(3.5)$ & $17.8(4.3)$ & $17.4(5.2)$ & $\operatorname{Chi}^{2}(2)=9.510, p=0.009$ & $z=-3.046, p=0.007, r=0.354^{a}$ \\
\hline \multicolumn{6}{|l|}{ Single task, double support } \\
\hline $\begin{array}{l}\text { Double support, } \% \text { of stride } \\
\text { time }(n=89)\end{array}$ & $36.2(6.9)$ & $37.4(7.3)$ & $47.3(10.4)$ & $\begin{array}{l}\mathrm{F}(2,86)=7.721, p=0.001 \\
\eta_{\mathrm{p}}^{2}=0.152\end{array}$ & $\begin{array}{l}p=0.001, M D=11.09, C_{95}[4.13, \\
18.05]^{b} \\
p=0.001, M D=9.89, C_{95}[3.41, \\
16.37]^{c}\end{array}$ \\
\hline Modified 30s CST $(n=77)$ & $7.5(3.3)$ & $9.0(3.7)$ & $4.8(1.7)$ & $\begin{array}{l}\mathrm{F}(2,74)=4.508, p=0.014 \\
\eta_{p}^{2}=0.109\end{array}$ & $\begin{array}{l}p=0.020, M D=-4.14, C_{95}[-7.73, \\
-0.55]^{c}\end{array}$ \\
\hline MMSE $(n=89)$ & $15.1(3.9)$ & $17.9(4.3)$ & $16.3(5.4)$ & $\operatorname{Chi}^{2}(2)=6.742, p=0.034$ & $z=-2.558, p=0.032, r=0.286^{a}$ \\
\hline \multicolumn{6}{|c|}{ Dual task, counting backwards, walking speed } \\
\hline Walking speed, $\mathrm{m} / \mathrm{sec}(\mathrm{n}=62)$ & $0.63(0.17)$ & $0.57(0.11)$ & $0.47(0.15)$ & $\begin{array}{l}F(2,59)=6.336, p=0.003 \\
\eta_{p}^{2}=0.177\end{array}$ & $\begin{array}{l}\mathrm{p}=0.001, \mathrm{MD}=-15.08, \mathrm{Cl}_{95}[-25.35, \\
-4.81]^{\mathrm{b}}\end{array}$ \\
\hline \multicolumn{6}{|c|}{ Dual task, counting backwards, stride length } \\
\hline \multicolumn{6}{|c|}{ No statistically significant differences } \\
\hline \multicolumn{6}{|c|}{ Dual task, counting backwards, double support } \\
\hline $\begin{array}{l}\text { Double support, } \% \text { of stride } \\
\text { time }(n=62)\end{array}$ & $37.7(9.2)$ & $40.0(7.1)$ & $47.8(12.9)$ & $\operatorname{Chi}^{2}(2)=6.496, p=0.039$ & $z=-2.532, p=0.034, r=0.479^{b}$ \\
\hline \multicolumn{6}{|c|}{ Dual task, naming animals, walking speed } \\
\hline Walking speed, $\mathrm{m} / \mathrm{sec}(\mathrm{n}=61)$ & $0.52(0.11)$ & $0.47(0.13)$ & $0.36(0.12)$ & $\begin{array}{l}F(2,58)=9.917, p<0.001 \\
\eta_{p}^{2}=0.255\end{array}$ & $\begin{array}{l}\mathrm{p}<0.001, \mathrm{MD}=-16.40, \mathrm{Cl}_{95}[-25.44, \\
-7.35]]^{\mathrm{b}}\end{array}$ \\
\hline TUG, sec $(n=61)$ & $18.8(9.2)$ & $20.8(16.9)$ & $24.8(12.1)$ & $\operatorname{Chi}^{2}(2)=6.360, p=0.042$ & n.s. \\
\hline \multicolumn{6}{|c|}{ Dual task, naming animals, stride length } \\
\hline Stride length, $\mathrm{cm}(n=61)$ & $77.9(14.0)$ & $71.8(18.3)$ & $61.6(18.3)$ & $\begin{array}{l}F(2,58)=3.596, p=0.034 \\
\eta_{p}^{2}=0.110\end{array}$ & $\begin{array}{l}p=0.031, \mathrm{MD}=-16.36, \mathrm{Cl}_{95}[-31.46, \\
-1.26]^{\mathrm{b}}\end{array}$ \\
\hline Walking aid, \% $(n=61)$ & $85.7 \%$ & $50.0 \%$ & $82.4 \%$ & $\mathrm{Chi}^{2}=7.540, \mathrm{p}=0.020$ & \\
\hline \multicolumn{6}{|c|}{ Dual task, naming animals, double support } \\
\hline $\begin{array}{l}\text { Double support, } \% \text { of stride } \\
\text { time }(n=61)\end{array}$ & $41.9(8.6)$ & $44.7(7.9)$ & $53.2(10.2)$ & $\begin{array}{l}F(2,61)=6.570, p=0.003 \\
\eta_{p}^{2}=0.185\end{array}$ & $\begin{array}{l}p=0.003, M D=11.35, C_{95}[3.38, \\
19.32]^{b} \\
p=0.010, M D=8.55, C_{95}[1.79, \\
15.30]^{c}\end{array}$ \\
\hline
\end{tabular}

30s CST: 30-s chair stand test, $\mathrm{Cl}_{95}$ : 95\% confidence interval, $d f$ : degrees of freedom, MD: mean difference, MMSE: Mini-Mental State Examination, $n$ :number, $n$. s.: not significant, SD: standard deviation, TUG: Timed Up \& Go Test

a post-hoc analysis: statistically significant better performance of non- compared to negative responders

${ }^{b}$ post-hoc analysis: statistically significant worse performance of positive compared to negative responders

c post-hoc analysis: statistically significant worse performance of positive compared to non-responders

often not possible due to our principle of ensuring the safety of participants at all times during the MEP. Additionally, we assumed that tasks aiming to improve balance, mobility, strength and function of lower limbs may be sufficient to positively affect gait performance [75-
78]. However, based on our findings, this assumption could not be confirmed. Thus, including a sufficient amount of specific walking exercises should be ensured in future physical activity interventions that aim at improving gait performance. 
Table 6 Impacts of changes in underlying motor and cognitive performance on changes of gait performance (intervention group, per protocol analysis)

\begin{tabular}{|c|c|c|c|c|c|c|c|c|c|}
\hline & $\mathrm{B}$ & SE & $\beta$ & $\mathrm{t}$ & $p$ & $R^{2}$ & Adjusted $R^{2}$ & $\mathrm{~F}\left(\mathrm{df}_{\text {numerator, }} \mathrm{df}_{\text {denominator }}\right), \mathrm{p}$ & $f^{2}$ \\
\hline \multicolumn{10}{|c|}{ Single task, changes in walking speed $(n=51)$} \\
\hline Model & & & & & & 0.207 & 0.191 & $F(1,49)=12.826, p=0.001$ & 0.24 \\
\hline Constant & -1.082 & 1.696 & & -0.638 & 0.526 & & & & \\
\hline Changes in TUG & -0.915 & 0.256 & -0.455 & -3.581 & 0.001 & & & & \\
\hline \multicolumn{10}{|l|}{ Single task, changes in stride length $(n=51)$} \\
\hline Model & & & & & & 0.146 & 0.128 & $F(1,49)=8.352, p=0.006$ & 0.15 \\
\hline Constant & -2.774 & 1.518 & & -1.828 & 0.074 & & & & \\
\hline Changes in modified SPPB & 2.107 & 0.729 & 0.382 & 2.890 & 0.006 & & & & \\
\hline \multicolumn{10}{|c|}{ Single task, changes in double support $(n=51)$} \\
\hline Model & & & & & & 0.144 & 0.126 & $F(1,49)=8.210, p=0.006$ & 0.14 \\
\hline Constant & 1.218 & 0.520 & & 2.341 & 0.023 & & & & \\
\hline Changes in modified SPPB & -0.716 & 0.250 & -0.379 & -2.865 & 0.006 & & & & \\
\hline \multicolumn{10}{|c|}{ Dual task, counting backwards, changes in walking speed $(n=42)$} \\
\hline Model & & & & & & 0.387 & 0.356 & $F(2,39)=12.322, p<0.001$ & 0.55 \\
\hline Constant & -5.331 & 1.995 & & -2.728 & 0.010 & & & & \\
\hline Changes in Clock Drawing Test & 3.597 & 0.961 & 0.474 & 3.742 & 0.001 & & & & \\
\hline Changes in modified 30s CST & 2.881 & 0.765 & 0.477 & 3.767 & 0.001 & & & & \\
\hline \multicolumn{10}{|c|}{ Dual task, counting backwards, changes in stride length $(n=42)$} \\
\hline Model & & & & & & 0.334 & 0.300 & $F(2,39)=9.771, p<0.001$ & 0.43 \\
\hline Constant & -0.661 & 1.626 & & -0.406 & 0.687 & & & & \\
\hline Changes in modified SPPB & 2.117 & 0.875 & 0.359 & 2.420 & 0.020 & & & & \\
\hline Changes in modified 30s CST & 1.519 & 0.716 & 0.314 & 2.122 & 0.040 & & & & \\
\hline \multicolumn{10}{|c|}{ Dual task, counting backwards, changes in double support $(n=42)$} \\
\hline Model & & & & & & 0.438 & 0.394 & $F(3,38)=9.871, p<0.001$ & 0.65 \\
\hline Constant & 2.449 & 0.654 & & 3.745 & 0.001 & & & & \\
\hline Changes in Clock Drawing Test & -1.167 & 0.306 & -0.469 & -3.814 & $<0.001$ & & & & \\
\hline Changes in modified 30s CST & -0.934 & 0.244 & -0.472 & -3.834 & $<0.001$ & & & & \\
\hline Changes in Digit Span backward & -0.682 & 0.333 & -0.250 & -2.052 & 0.047 & & & & \\
\hline \multicolumn{10}{|c|}{ Dual task, naming animals, changes in walking speed $(n=40)$} \\
\hline Model & & & & & & 0.280 & 0.241 & $F(2,37)=7.184, p=0.002$ & 0.31 \\
\hline Constant & -1.991 & 1.823 & & -1.092 & 0.282 & & & & \\
\hline Changes in modified 30s CST & 1.796 & 0.706 & 0.364 & 2.544 & 0.015 & & & & \\
\hline Changes in Trail Making Test & 0.501 & 0.228 & 0.313 & 2.192 & 0.035 & & & & \\
\hline \multicolumn{10}{|c|}{ Dual task, naming animals, changes in stride length $(n=40)$} \\
\hline Model & & & & & & 0.296 & 0.258 & $F(2,37)=7.788, p=0.002$ & 0.35 \\
\hline Constant & -0.360 & 1.681 & & -0.214 & 0.832 & & & & \\
\hline Changes in modified SPPB & 2.790 & 0.781 & 0.500 & 3.571 & 0.001 & & & & \\
\hline Changes in Clock Drawing Test & -1.774 & 0.785 & -0.316 & -2.259 & 0.030 & & & & \\
\hline \multicolumn{10}{|c|}{ Dual task, naming animals, changes in double support $(n=40)$} \\
\hline Model & & & & & & 0.198 & 0.177 & $F(1,38)=9.378, p=0.004$ & 0.22 \\
\hline Constant & 1.426 & 0.927 & & 1.538 & 0.132 & & & & \\
\hline Changes in Digit Span forward & -1.736 & 0.567 & -0.445 & -3.062 & 0.004 & & & & \\
\hline
\end{tabular}


Differences in characteristics between positive, negative, and non-responders and impact of changes in underlying motor and cognitive performance on changes in gait performance

Despite not having observed positive overall effects, additional analyses showed that between 61 and $81 \%$ of participants in the IG improved or maintained their gait performance after participating in the MEP. In studies among IWD, who usually experience rapid decline of motor, cognitive, and gait performance [8], even maintaining the current levels of performance is indicative of a beneficial effect. In order to better understand the prerequisites and impacts to induce such benefits from physical activity interventions, we conducted secondary analyses that focused on examining differences of baseline performance and sample characteristics between positive, non-, and negative responders, and also considered impacts of underlying changes in motor and cognitive performance on changes in gait performance.

As compared to negative and non-responders, positive responders primarily showed lower gait performance at baseline and additionally demonstrated lower performance in single motor assessments. Moreover, nonresponders were less cognitively impaired than negative responders. Accordingly, low motor and gait performance as well as increased cognitive performance appear to be prerequisites for IWD in order to benefit from the MEP. Additionally, stepwise regression analyses supported the hypothesis that changes in underlying motor and cognitive performance have an impact on changes in gait performance. Indeed, the respective statistical models explained between 12.6 and $39.4 \%$ of the overall variance.

Focusing on prerequisites related to the effectiveness of the MEP, the observed lower motor performance of positive responders compared to non- and negative responders at baseline may indicate a greater potential for performance improvements for participants who enter the intervention with lower baseline levels of motor performance. As described above, it was not always possible to include more complex walking tasks throughout our intervention. Accordingly, the requirements necessary to induce improvements may not have reached critical thresholds in all participants. Moreover, our findings support the assumption that IWD must have sufficient cognitive capacities in order for them to successfully participate in physical activity interventions. In contrast, severe cognitive impairments may affect IWD in following instructions or adequately performing exercise tasks. Individuals with more severe cognitive impairments may depend even more on specific didactic concepts.

Surprisingly, we observed a statistically significant higher cognitive performance only among nonresponders and in single task conditions. Positive responders also showed higher cognitive performance than negative responders, albeit not reaching statistical significance possibly due to a relatively lower number of positive responders. When we compared cognitive performance of participants in single and dual task conditions, we observed that participants with more severe cognitive impairments were less likely to successfully perform the walking with additional dual tasks (single task: $\mathrm{MMSE}=16.9$ (4.5), 45\% with MMSE < 17; dual task: $\mathrm{MMSE}=18.4$ (4.0)/17.8 (4.3) 29\%/26\% with MMSE $<17)$. Accordingly, cognitive performance of participants who completed dual task conditions was more consistent and this may explain why cognitive performance did not differ between positive, non-, and negative responders.

Stepwise regression analyses showed differential impacts of changes in underlying motor and cognitive performance, depending on spatiotemporal gait parameter and walking condition. As expected, improvements in gait performance were associated with improvements in underlying motor and cognitive performance. The observed opposite relation between stride length in dual task naming animals condition and the Clock Drawing Test requires further examination. The amount of explained variance was higher for dual task than single task conditions. In dual task conditions, changes in motor and cognitive performance were statistically significant predictors, while gait parameters in the single task condition were only affected by motor predictors. Accordingly, changes in cognitive performance may be particularly required for changes in dual task conditions, which are primarily determined by motor and cognitive demands. Dual task performance while walking is highly relevant with regard to fall prevention, and worse performance is associated with increased risk of falls [13]. Thus, fall prevention interventions should consider dual tasks and include both, motor and cognitive exercises.

At the motor level, changes in strength and function of lower limbs as well as mobility were statistically significant predictors. The related performance was assessed with modified 30s CST, modified SPPB, which considers balance, mobility, and strength, and TUG. These findings indicate that there are several motor impacts related to changes in gait performance, and further emphasize the importance of multimodal interventions. Unexpectedly, changes in balance performance were not a statistically significant predictor. However, we assessed balance only in static positions, which may have different demands as compared to dynamic balance conditions while walking [79, 80]. Moreover, the frequent use of walking aids may have eliminated the potential impact of changes in balance performance [81]. Assumptions at the cognitive level could not be made, as cognitive predictors differed across established regression models. 


\section{Comparison with previous studies}

The findings of this randomized controlled trial are not fully in line with those observed in previous studies. In contrast to previous studies, which predominantly reported positive effects for stride length and stride time in single and dual task conditions [39-46], our investigation did not confirm the effectiveness of an MEP for these spatiotemporal gait parameters. In accordance with 20 previous studies, we did not observe statistically significant effects on walking speed [24-38, 42, 44, 47, 48] and percent of double support [44, 45], while twelve others did for single [17-23, 40, 41, 43, 46] and dual task conditions [28].

These inconsistent findings may be related to different study designs, gait assessments, interventions, and sample characteristics between previous research and our study. For example, studies considering walking speed in single task condition and reporting positive effects are characterized by more walking tasks included in the intervention [17, 19-21, 23, 40, 41, 43], higher exercise intensities [19, 20, 43], participants with less cognitive impairments [19, 21, 43], worse baseline walking performance $[17,18,22,23,46]$, better baseline walking performance $[19,20,43]$, as well as assessing gait performance during fast walking [27, 29, 31, 48] compared to our study. While the first four observations reflect the results and indications of this study, better baseline walking performance may enable instructors to implement higher exercise intensities and larger amounts of specific walking tasks. Moreover, fast walking speed may be a more specific indicator for changes. However, these indications need to be considered with caution, as several impacts and the heterogeneity of previous studies hamper comparisons.

Heterogeneity is a challenge also occurring in other studies. Some differences between our study and studies showing positive effects (considering walking speed in single task condition) may be due to less cognitive impairments of participants [19, 21, 43], only including participants able to walk without walking aids [19, 40, $43,46]$, hints of enhanced individual supervision [20, 21, $23,40,41,46]$, or classification in homogeneous subgroups [17]. One may argue that the negative impact of heterogeneity is less critical in groups of participants in mild stages of dementia, as these participants are rather able to implement different instructions. Moreover, it can be assumed that participants not dependent on walking aids are less likely to fall. Accordingly, it is possible to apply the same exercise for those participants, even if they differ in their walking performance. In contrast, participants with enhanced fall risks rely on several modifications due to safety reasons. Besides these differences related to sample characteristics, an enhanced individual supervision allows the individualization of exercise tasks and adapted support. However, such concepts cannot always be transferred into practice because of limited personal resources. Accordingly, new approaches feasible in group settings are necessary, such as the classification of homogeneous subgroups.

In addition, the etiology or underlying causes of dementia, as well as frequency and duration of physical activity interventions differ considerably among previous studies and make a comparison somewhat difficult. For example, previous studies have included participants with $\mathrm{AD}$, whereas other have included individuals with mixed etiologies or have not reported etiologies. Due to differences in underlying pathologic changes, various etiologies may have a differential impact on the effects of physical activity on motor or cognitive performance. In addition, duration and frequency of physical activity interventions in previous studies vary between 3 weeks and 36 months, with sessions carried out once per week to twice daily. However, most studies applied a protocol consisting of two to three sessions per week for at least 12 weeks. This is in line with recommendations of recent systematic reviews and seems to be most effective in eliciting any effects on motor and cognitive performance in IWD [82-86]. Accordingly, our intervention had a duration of 16 weeks with a frequency of two sessions per week.

To the best of our knowledge, there are no published studies that compared the characteristics of positive, non-, and negative responders or investigated impacts of changes in underlying motor and cognitive performance on changes in gait performance.

\section{Strengths and limitations}

With this multicenter randomized controlled trial, we aimed at conducting high-quality research to investigate the effectiveness of a physical activity intervention on gait performance in IWD. The strengths of the study include the emphasis on high-quality methods and a detailed reporting of our methods and findings [53]. Of note, we had a large sample size of over 300 individuals with mild to moderate dementia, our assessments were deemed adequate for IWD by an expert panel [87], and our MEP was specifically tailored to fit the needs and characteristics of IWD (please refer to [53]).

Nevertheless, several limitations pertain. First, multimodal interventions do not allow to unambiguously draw conclusions about causality, i.e. observed effects may be related to the MEP itself, but could also be due to the group setting and thus enhanced social interaction, or additional attention that participants received from the exercise instructors. Therfore, additional control conditions such as non-exercise groups that engage in mentally stimulating or social activities such as singing or playing cards together could have helped to limit 
this potential bias. Second, insufficient intensity and specificity may be potential limitations of the MEP related to the primary aim to improve spatiotemporal gait parameters. Several factors, such as characteristics of participants, group setting, or field conditions did not allow us to reach higher intensity levels, a larger amount of specific walking tasks, and progression of the MEP as initially planned. Importantly, the MEP was mainly performed in a seated position which may have limited impact on gait performance albeit we chose exercises with medium to submaximal intensity. One potential strategy to overcome this limitation is to conduct exercise interventions with smaller groups to ensure safety of participants while reaching higher intensities at the same time. In line with this, the secondary analysis showed that participants who benefitted had a lower motor baseline performance. Future interventions need to better consider individual prerequisites of participants and accordingly adapt intensity, specificity, as well as progression of exercises. Third, the assessments to determine motor performance used in this study are widely used in research but have not been specifically developed for IWD. Even though we intensively discussed the adequateness of these assessments during an expert panel [87] and carefully selected the most appropriate ones, we cannot rule out the possibility that the use of existing assessments not specifically designed for IWD may have led to biased results. For example, these assessments often do not sufficiently take into account fluctuating daily forms and motivational aspects that may play a role when examining IWD. Accordingly, results could reflect unfavorable conditions, reduced motivation, or lack of interest instead of actual motor performance. Therefore, it is critically important for future research to explore tailored motor assessments for use in IWD. Finally, our study sample included participants that did not have a confirmed diagnosis of dementia, in most cases due to limited financial resources or access to diagnostic tools and assessments. This may have an impact on the interpretability of findings.

\section{Conclusions}

This multicenter randomized controlled trial contributes to the growing body of literature that aims at improving physical activity interventions for IWD. It shows that one standardized MEP is not effective in reducing the decline in gait performance among IWD in general. However, several participants of the IG were able to improve or maintain their gait performance after undergoing the MEP. Moreover, findings of secondary analyses allow for drawing conclusions on prerequisites and required changes that may be necessary for IWD to benefit from the MEP. These factors have important implications and should thus be considered when establishing future physical activity interventions.

Our main conclusion is that it is essential to develop and provide individualized physical activity interventions for IWD, and to consider individual characteristics and needs to improve effectiveness rather than having one standardized physical activity intervention; albeit acknowledging that this is currently more applicable to a research setting and probably more challenging for clinical settings such as nursing homes. Based on observed results in responder-non-responder-analyses, we suggest tailoring physical activity interventions to baseline performance of intended outcomes and severity of cognitive impairment. To this end, we here provide preliminary criteria on how to tailor physical activity interventions to fit the specific needs of IWD. However, further investigation and refinement of these criteria is needed to better characterize different clusters of IWD. Aside from individual characteristics and needs, it is also important to consider intended purposes when establishing physical activity interventions.

Our findings indicate that physical activity interventions aiming to improve gait performance in IWD should include multimodal motor exercises (e.g. walking, strength, balance, and mobility). As changes in motor and cognitive performance are statistically significant independent predictors for changes in gait performance, both motor and cognitive tasks should be included in interventions to potentially increase the beneficial effects on gait performance and fall prevention. Linking both conclusions, individualized approaches, which include relevant contributors for improving intended outcomes, while also tailoring requirements to prerequisites and focusing on those exercises in order to improve outcomes of especially low capacity, seem to be most promising for improving the effectiveness of physical activity interventions in IWD.

\section{Supplementary information}

Supplementary information accompanies this paper at https://doi.org/10. 1186/s12877-020-01635-3.

Additional file 1.

Additional file 2.

Additional file 3.

Additional file 4.
Abbreviations

30s CST: 30-s chair stand test; ANOVA: Analysis of variance; CG: Control group; CIRS: Cumulative IIIness Rating Scale; IG: Intervention group;

IWD: Individuals with dementia; MEP: Multimodal exercise program; MMSE: Mini-Mental State Examination; SPPB: Short Physical Performance Battery; TUG: Timed Up \& Go Test 


\section{Acknowledgments}

We are grateful to all participants of the study and members of our research team. We would like to thank Luisa Appelles for the coordination of fieldwork and for entering data, Bastian Anedda for establishing a script to process spatiotemporal gait parameters, Anela Hadzic for supporting the preparation of gait data, and Professor Darko Jekauc for his advice on the publishing process. We acknowledge support by the KIT-Publication Fund of the Karlsruhe Institute of Technology.

\section{Authors' contributions}

All authors were members of the research team and participated in the implementation of the study. AW conceived the idea of this study, along with ST and AS. All authors contributed to the conception and design of the study. ST coordinated the study under the direct supervision of AW. ST and AS were responsible for the implementation of the study. AS developed und supervised the multimodal exercise program. ST, BB, SR, and TS intensively discussed and established the approach to process and analyze spatiotemporal gait parameters. ST performed the statistical analysis. ST, BB, and JK interpreted the data. All authors were involved in planning and writing the manuscript. ST wrote the manuscript. All authors provided critical feedback and approved the final manuscript.

\section{Funding}

This project is financially supported by the Dietmar Hopp Stiftung (St. LeonRot, Germany). The sponsor did not have any role in the design of the study, neither in its execution, the collection, analysis or interpretation of data, the decision to submit results nor in writing the report.

\section{Availability of data and materials}

Due to data protection reasons of the Karlsruhe Institute of Technology (Karlsruhe, Germany), restrictions may apply to the availability of these data. If permissible, the datasets used and/or analyzed during the current study are available from the corresponding author on reasonable request.

\section{Ethics approval and consent to participate}

The ethics committee of the Karlsruhe Institute of Technology (Karlsruhe, Germany) granted ethical approval. Furthermore, we obtained written informed consent prior to the study from all participants or their legal guardians, respectively.

\section{Consent for publication}

Not applicable.

\section{Competing interests}

The authors declare that they have no competing interests.

\section{Author details}

'Karlsruhe Institute of Technology, Institute of Sports and Sports Science, Engler-Bunte-Ring 15, 76131 Karlsruhe, Germany. ${ }^{2}$ Department of Sport and Sport Science, University of Freiburg, Freiburg, Germany. ${ }^{3}$ Department of Health Sciences Research, Mayo Clinic, Rochester, MN, USA.

\section{Received: 12 February 2020 Accepted: 29 June 2020}

\section{Published online: 16 July 2020}

\section{References}

1. Valkanova V, Ebmeier KP. What can gait tell us about dementia? Review of epidemiological and neuropsychological evidence. Gait Posture. 2017;53: 215-23. https://doi.org/10.1016/j.gaitpost.2017.01.024

2. Mahlknecht P, Kiechl S, Bloem BR, Willeit J, Scherfler C, Gasperi A, et al. Prevalence and burden of gait disorders in elderly men and women aged 60-97 years: a population-based study. PLoS One. 2013;8:e69627. https://doi. org/10.1371/journal.pone.0069627.

3. Mc Ardle R, Morris R, Wilson J, Galna B, Thomas AJ, Rochester L. What can quantitative gait analysis tell us about dementia and its subtypes? A structured review. J Alzheimers Dis. 2017;60:1295-312. https://doi.org/10. 3233/JAD-170541.

4. van lersel MB, Hoefsloot W, Munneke M, Bloem BR, Olde Rikkert MGM. Systematic review of quantitative clinical gait analysis in patients with dementia. Z Gerontol Geriatr. 2004;37:27-32. https://doi.org/10.1007/s00391004-0176-7.
5. Allali G, Verghese J. Management of gait changes and fall risk in $\mathrm{MCl}$ and dementia. Curr Treat Options Neurol. 2017;19:29. https://doi.org/10.1007/ s11940-017-0466-1.

6. Allan LM, Ballard CG, Burn DJ, Kenny RA. Prevalence and severity of gait disorders in Alzheimer's and non-Alzheimer's dementias. J Am Geriatr Soc. 2005;53:1681-7. https://doi.org/10.1111/j.1532-5415.2005.53552.x.

7. World Health Organization. Global action plan on the public health response to dementia 2017-2025. 2017. https://apps.who.int/iris/bitstream/ handle/10665/259615/9789241513487-eng.pdf;jsessionid=56E041EC9A4 ECD2FAB7A9DDAFE0D2525? sequence=1. Accessed 17 Apr 2020.

8. Alzheimer's Association. 2019 Alzheimer's disease facts and figures. Alzheimers Dement. 2019;15:321-87. https://doi.org/10.1016/j.jalz.2019.01.010.

9. Alexander NB, Goldberg A. Gait disorders: search for multiple causes. Cleve Clin J Med. 2005;72:586 589-90, 592-4 passim.

10. Hausdorff JM. Gait dynamics, fractals and falls: finding meaning in the stride-to-stride fluctuations of human walking. Hum Mov Sci. 2007;26:55589. https://doi.org/10.1016/j.humov.2007.05.003.

11. Amboni M, Barone P, Hausdorff JM. Cognitive contributions to gait and falls: evidence and implications. Mov Disord. 2013;28:1520-33. https://doi.org/10. 1002/mds.25674.

12. Stites SD, Harkins K, Rubright JD, Karlawish J. Relationships between cognitive complaints and quality of life in older adults with mild cognitive impairment, mild Alzheimer disease dementia, and normal cognition. Alzheimer Dis Assoc Disord. 2018;32:276-83. https://doi.org/10.1097/WAD. 0000000000000262

13. Montero-Odasso M, Verghese J, Beauchet O, Hausdorff JM. Gait and cognition: a complementary approach to understanding brain function and the risk of falling. J Am Geriatr Soc. 2012;60:2127-36. https://doi.org/10. 1111/j.1532-5415.2012.04209.x.

14. Segev-Jacubovski O, Herman T, Yogev-Seligmann G, Mirelman A, Giladi N, Hausdorff JM. The interplay between gait, falls and cognition: can cognitive therapy reduce fall risk? Expert Rev Neurother. 2011;11:1057-75. https://doi. org/10.1586/ern.11.69.

15. Cohen JA, Verghese J, Zwerling JL. Cognition and gait in older people. Maturitas. 2016;93:73-7. https://doi.org/10.1016/j.maturitas.2016.05.005.

16. Hortobágyi T, Lesinski M, Gäbler M, VanSwearingen JM, Malatesta $D_{\text {, }}$ Granacher U. Effects of three types of exercise interventions on healthy old adults' gait speed: a systematic review and meta-analysis. Sports Med. 2015; 45:1627-43. https://doi.org/10.1007/s40279-015-0371-2.

17. Rolland Y, Pillard F, Klapouszczak A, Reynish E, Thomas D, Andrieu S, et al. Exercise program for nursing home residents with Alzheimer's disease: a 1year randomized, controlled trial. J Am Geriatr Soc. 2007;55:158-65. https:// doi.org/10.1111/j.1532-5415.2007.01035.x.

18. Toulotte C, Fabre C, Dangremont B, Lensel G, Thévenon A. Effects of physical training on the physical capacity of frail, demented patients with a history of falling: a randomised controlled trial. Age Ageing. 2003;32:67-73.

19. Hauer K, Schwenk M, Zieschang T, Essig M, Becker C, Oster P. Physical training improves motor performance in people with dementia: a randomized controlled trial. J Am Geriatr Soc. 2012;60:8-15. https://doi.org/ 10.1111/j.1532-5415.2011.03778.x.

20. Bossers WJR, Scherder EJA, Boersma F, Hortobágyi T, van der Woude LHV, van Heuvelen MJG. Feasibility of a combined aerobic and strength training program and its effects on cognitive and physical function in institutionalized dementia patients. A pilot study. PLoS ONE. 2014;9:e97577. https://doi.org/10.1371/journal.pone.0097577.

21. Manckoundia P, Taroux M, Kubicki A, Mourey F. Impact of ambulatory physiotherapy on motor abilities of elderly subjects with Alzheimer's disease. Geriatr Gerontol Int. 2014;14:167-75. https:/doi.org/10.1111/ggi.12075.

22. Ahn N, Kim K. Effects of an elastic band resistance exercise program on lower extremity muscle strength and gait ability in patients with Alzheimer's disease. J Phys Ther Sci. 2015;27:1953-5. https://doi.org/10.1589/jpts.27.1953.

23. Aman $E$, Thomas DR. Supervised exercise to reduce agitation in severely cognitively impaired persons. J Am Med Dir Assoc. 2009;10:271-6. https:/ doi.org/10.1016/j.jamda.2008.12.053

24. Steinberg M, Leoutsakos J-MS, Podewils LJ, Lyketsos CG. Evaluation of a home-based exercise program in the treatment of Alzheimer's disease: the maximizing Independence in dementia (MIND) study. Int J Geriatr Psychiatry. 2009;24:680-5. https://doi.org/10.1002/gps.2175.

25. Telenius EW, Engedal K, Bergland A. Effect of a high-intensity exercise program on physical function and mental health in nursing home residents 
with dementia: an assessor blinded randomized controlled trial. PLoS One. 2015;10:e0126102. https://doi.org/10.1371/journal.pone.0126102.

26. Sobol NA, Hoffmann K, Frederiksen KS, Vogel A, Vestergaard K, Brændgaard $H$, et al. Effect of aerobic exercise on physical performance in patients with Alzheimer's disease. Alzheimers Dement. 2016;12:1207-15. https://doi.org/ 10.1016/j.jalz.2016.05.004

27. Hageman PA, Thomas VS. Gait performance in dementia: the effects of a 6week resistance training program in an adult day-care setting. Int J Geriatr Psychiatry. 2002;17:329-34. https://doi.org/10.1002/gps.597.

28. Tay L, Lim WS, Chan M, Ali N, Chong MS. A combined cognitive stimulation and physical exercise programme (MINDVital) in early dementia: differential effects on single- and dual-task gait performance. Gerontology. 2016;62: 604-10. https://doi.org/10.1159/000444084.

29. Thomas VS, Hageman PA. Can neuromuscular strength and function in people with dementia be rehabilitated using resistance-exercise training?: results from a preliminary intervention study. J Gerontol A Biol Sci Med Sci. 2003;58:M746-51. https://doi.org/10.1093/gerona/58.8.M746.

30. Bossers WJR, van der Woude LHV, Boersma F, Hortobágyi T, Scherder EJA, van Heuvelen MJG. A 9-week aerobic and strength training program improves cognitive and motor function in patients with dementia: a randomized, controlled trial. Am J Geriatr Psychiatry. 2015;23:1106-16. https://doi.org/10.1016/j.jagp.2014.12.191.

31. Dawson N, Judge KS, Gerhart H. Improved functional performance in individuals with dementia after a moderate-intensity home-based exercise program: a randomized controlled trial. J Geriatr Phys Ther. 2019;42:18-27. https://doi.org/10.1519/JPT.0000000000000128.

32. Hauer K, Ullrich P, Dutzi I, Beurskens R, Kern S, Bauer J, Schwenk M. Effects of standardized home training in patients with cognitive impairment following geriatric rehabilitation: a randomized controlled pilot study. Gerontology. 2017:63:495-506. https://doi.org/10.1159/000478263.

33. de Souto Barreto P, Cesari M, Denormandie P, Armaingaud D, Vellas B, Rolland $Y$. Exercise or social intervention for nursing home residents with dementia: a pilot randomized, controlled trial. J Am Geriatr Soc. 2017;65: E123-9. https://doi.org/10.1111/jgs.14947.

34. Toots $A$, Littbrand $H$, Holmberg $H$, Nordström $P$, Lundin-Olsson L, Gustafson $Y$, Rosendahl E. Walking aids moderate exercise effects on gait speed in people with dementia: a randomized controlled trial. J Am Med Dir Assoc. 2017;18:227-33. https://doi.org/10.1016/j.jamda.2016.09.003.

35. Cadore EL, Moneo ABB, Mensat MM, Muñoz AR, Casas-Herrero A, Rodriguez Mañas $L$, Izquierdo M. Positive effects of resistance training in frail elderly patients with dementia after long-term physical restraint. Age (Dordr). 2014; 36:801-11. https://doi.org/10.1007/s11357-013-9599-7.

36. Junge T, Knudsen HK, Kristensen HK. The effect of long-term, group-based physical, cognitive and social activities on physical performance in elderly, community-dwelling people with mild to moderate dementia. Dementia (Lond). 2018. https://doi.org/10.1177/1471301218806376.

37. McCaffrey R, Park J, Newman D, Hagen D. The effect of chair yoga in older adults with moderate and severe Alzheimer's disease. Res Gerontol Nurs. 2014:7:171-7. https://doi.org/10.3928/19404921-20140218-01.

38. Kuiack SL, Campbell WW, Evans WJ. A structured resistive training program improves muscle strength and power in elderly persons with dementia. Act Adapt Aging. 2004;28:35-47. https://doi.org/10.1300/J016v28n01_03.

39. Coelho FGDM, Andrade LP, Pedroso RV, Santos-Galduroz RF, Gobbi S, Costa $J$ LR, Gobbi LTB. Multimodal exercise intervention improves frontal cognitive functions and gait in Alzheimer's disease: a controlled trial. Geriatr Gerontol Int. 2013. https://doi.org/10.1111/j.1447-0594.2012.00887.x.

40. Kemoun G, Thibaud M, Roumagne N, Carette P, Albinet C, Toussaint L, et al. Effects of a physical training programme on cognitive function and walking efficiency in elderly persons with dementia. Dement Geriatr Cogn Disord. 2010;29:109-14. https://doi.org/10.1159/000272435.

41. Perrochon A, Tchalla AE, Bonis J, Perucaud F, Mandigout S. Effects of a multicomponent exercise program on spatiotemporal gait parameters, risk of falling and physical activity in dementia patients. Dement Geriatr Cogn Dis Extra. 2015;5:350-60. https://doi.org/10.1159/000435772.

42. Schwenk M, Dutzi I, Englert S, Micol W, Najafi B, Mohler J, Hauer K. An intensive exercise program improves motor performances in patients with dementia: translational model of geriatric rehabilitation. J Alzheimers Dis. 2014:39:487-98. https://doi.org/10.3233/JAD-130470.

43. Schwenk M, Zieschang T, Englert S, Grewal G, Najafi B, Hauer K. Improvements in gait characteristics after intensive resistance and functional training in people with dementia: a randomised controlled trial. BMC Geriatr. 2014;14:73. https://doi.org/10.1186/1471-2318-14-73.

44. Pedrinolla A, Venturelli M, Fonte C, Munari D, Benetti MV, Rudi D, et al. Exercise training on locomotion in patients with Alzheimer's disease: a feasibility study. J Alzheimers Dis. 2018;61:1599-609. https://doi.org/10.3233/ JAD-170625.

45. Orcioli-Silva D, Barbieri FA, Simieli L, Santos PCR, Beretta VS, Coelho FGDM, et al. A program of physical activity improves gait impairment in people with Alzheimer's disease. Motriz. 2018;24:452. https://doi.org/10.1590/s19806574201800010009.

46. Kim J-S, Kang M-H, Moon D-C, Oh J-S. Effect of pelvic tilt exercise using pressure-based visual biofeedback training on the gait parameter in elderly patients with Alzheimer's disease. Eur Geriatr Med. 2017;8:30-6. https://doi. org/10.1016/j.eurger.2016.11.001.

47. Suttanon P, Hill KD, Said CM, Williams SB, Byrne KN, LoGiudice D, et al. Feasibility, safety and preliminary evidence of the effectiveness of a homebased exercise programme for older people with Alzheimer's disease: a pilot randomized controlled trial. Clin Rehabil. 2013;27:427-38. https://doi. org/10.1177/0269215512460877.

48. Ries JD, Hutson J, Maralit LA, Brown MB. Group balance training specifically designed for individuals with Alzheimer disease: impact on berg balance scale, timed up and go, gait speed, and mini-mental status examination. J Geriatr Phys Ther. 2015;38:183-93. https://doi.org/10.1519/JPT. 0000000000000030 .

49. Yogev-Seligmann G, Hausdorff JM, Giladi N. The role of executive function and attention in gait. Mov Disord. 2008;23:329-42; quiz 472. https://doi.org/ 10.1002/mds.21720.

50. Sheridan PL, Hausdorff JM. The role of higher-level cognitive function in gait: executive dysfunction contributes to fall risk in Alzheimer's disease. Dement Geriatr Cogn Disord. 2007;24:125-37. https://doi.org/10.1159/ 000105126.

51. Moher D, Hopewell S, Schulz KF, Montori V, Gøtzsche PC, Devereaux PJ et al. CONSORT 2010 explanation and elaboration: updated guidelines for reporting parallel group randomised trials. BMJ. 2010;340:C869. https://doi. org/10.1136/bmj.c869.

52. Schulz KF, Altman DG, Moher D. CONSORT 2010 statement: updated guidelines for reporting parallel group randomized trials. Ann Intern Med. 2010;152:726-32. https://doi.org/10.7326/0003-4819-152-11-20100601000232.

53. Trautwein S, Scharpf A, Barisch-Fritz B, Niermann C, Woll A. Effectiveness of a 16-week multimodal exercise program on individuals with dementia: study protocol for a multicenter randomized controlled trial. JMIR Res Protoc. 2017;6:e35. https://doi.org/10.2196/resprot.6792.

54. Saghaei M, Saghaei S. Implementation of an open-source customizable minimization program for allocation of patients to parallel groups in clinical trials. J Biomed Sci Eng. 2011;4:734-9. https://doi.org/10.4236/jbise.2011. 411090.

55. Faul F, Erdfelder E, Lang A-G, Buchner A. G*power 3: a flexible statistical power analysis program for the social, behavioral, and biomedical sciences. Behav Res Methods. 2007;39:175-91. https://doi.org/10.3758/BF03193146.

56. Linn BS, Linn MW, Gurel LEE. Cumulative illness rating scale. J Am Geriatr Soc. 1968;16:622-6. https://doi.org/10.1111/j.1532-5415.1968.tb02103.x.

57. Ries JD, Echternach JL, Nof L, Gagnon Blodgett M. Test-retest reliability and minimal detectable change scores for the timed "up \& go" test, the sixminute walk test, and gait speed in people with Alzheimer disease. Phys Ther. 2009;89:569-79. https://doi.org/10.2522/ptj.20080258.

58. Wittwer JE, Webster KE, Andrews PT, Menz HB. Test-retest reliability of spatial and temporal gait parameters of people with Alzheimer's disease. Gait Posture. 2008;28:392-6. https://doi.org/10.1016/j.gaitpost.2008.01.007.

59. Kressig RW, Beauchet O. Guidelines for clinical applications of spatiotemporal gait analysis in older adults. Aging Clin Exp Res. 2006;18:174-6. https://doi.org/10.1007/BF03327437.

60. Beauchet O, Allali G, Sekhon H, Verghese J, Guilain S, Steinmetz J-P, et al. Guidelines for assessment of gait and reference values for spatiotemporal gait parameters in older adults: the biomathics and Canadian gait consortiums initiative. Front Hum Neurosci. 2017;11:353. https://doi.org/10. 3389/fnhum.2017.00353.

61. Schwenk M, Zieschang T, Oster P, Hauer K. Dual-task performances can be improved in patients with dementia: a randomized controlled trial. Neurology. 2010;74:1961-8. https://doi.org/10.1212/WNL.0b013e3181e39696. 
62. Abernethy B. Dual-task methodology and motor skills research: some applications and methodological constraints. J Hum Mov Stud. 1988;14:101-32

63. Rossiter-Fornoff JE, Wolf SL, Wolfson LI, Buchner DM. A cross-sectional validation study of the FICSIT common data base static balance measures. Frailty and injuries: cooperative studies of intervention techniques. J Gerontol A Biol Sci Med Sci. 1995;50:M291-7. https://doi.org/10.1093/ gerona/50A.6.M291.

64. Podsiadlo D, Richardson S. The timed "up \& go": a test of basic functional mobility for frail elderly persons: a test of basic functional mobility for frail elderly persons. J Am Geriatr Soc. 1991;39:142-8. https://doi.org/10.1111/j. 1532-5415.1991.tb01616.x.

65. Jones CJ, Rikli RE, Beam WC. A 30-s chair-stand test as a measure of lower body strength in community-residing older adults. Res Q Exerc Sport. 1999; 70:113-9. https://doi.org/10.1080/02701367.1999.10608028.

66. Blankevoort CG, Van Heuvelen MJG, Scherder EJA. Reliability of six physical performance tests in older people with dementia. Phys Ther. 2013;93:69-78. https://doi.org/10.2522/ptj.20110164.

67. Guralnik JM, Simonsick EM, Ferrucci L, Glynn RJ, Berkman LF, Blazer DG, et al. A short physical performance battery assessing lower extremity function: association with self-reported disability and prediction of mortality and nursing home admission. J Gerontol. 1994;49:M85-94. https://doi.org/ 10.1093/geronj/49.2.M85.

68. Folstein MF, Folstein SE, McHugh PR. "Mini-mental state". A practical method for grading the cognitive state of patients for the clinician. J Psychiatr Res. 1975;12:189-98. https://doi.org/10.1016/0022-3956(75)90026-6.

69. Shulman Kl, Shedletsky R, Silver IL. The challenge of time: clock-drawing and cognitive function in the elderly. Int J Geriatr Psychiatry. 1986;1:135-40. https://doi.org/10.1002/gps.930010209.

70. Mendes-Santos LC, Mograbi D, Spenciere B, Charchat-Fichman H. Specific algorithm method of scoring the clock drawing test applied in cognitively normal elderly. Dement Neuropsychol. 2015;9:128-35. https://doi.org/10. 1590/1980-57642015DN92000007.

71. Reitan RM. Trail making test: manual for administration and scoring; 1992.

72. Reitan RM. Validity of the trail making test as an indicator of organic brain damage. Percept Mot Skills. 1958:8:271-6. https://doi.org/10.2466/pms.1958. 8.3.271.

73. Wilde NJ, Strauss E, Tulsky DS. Memory span on the Wechsler scales. J Clin Exp Neuropsychol. 2004;26:539-49. https://doi.org/10.1080/ 13803390490496605.

74. Trautwein S, Maurus P, Barisch-Fritz B, Hadzic A, Woll A. Recommended motor assessments based on psychometric properties in individuals with dementia: a systematic review. Eur Rev Aging Phys Act. 2019;16:876. https:// doi.org/10.1186/s11556-019-0228-z.

75. McGough EL, Logsdon RG, Kelly VE, Teri L. Functional mobility limitations and falls in assisted living residents with dementia: physical performance assessment and quantitative gait analysis. J Geriatr Phys Ther. 2013;36:78-86. https://doi.org/10.1519/JPT.0b013e318268de7f.

76. Nakayama N, Suzuki M, Hamaguchi T. Relationship between knee extension strength and gait styles in patients with dementia. Medicine (Baltimore). 2019;98:e14958. https://doi.org/10.1097/MD.0000000000014958.

77. Suzuki M, Kirimoto $H$, Inamura A, Yagi M, Omori Y, Yamada S. The relationship between knee extension strength and lower extremity functions in nursing home residents with dementia. Disabil Rehabil. 2012;34: 202-9. https://doi.org/10.3109/09638288.2011.593678.

78. Suzuki M, Yamada S, Inamura A, Omori Y, Kirimoto H, Sugimura S, Miyamoto M. Reliability and validity of measurements of knee extension strength obtained from nursing home residents with dementia. Am J Phys Med Rehabil. 2009;88:924-33. https://doi.org/10.1097/PHM.0b013e3181ae1003.

79. Granacher U, Bridenbaugh SA, Muehlbauer T, Wehrle A, Kressig RW. Agerelated effects on postural control under multi-task conditions. Gerontology. 2011;57:247-55. https://doi.org/10.1159/000322196.

80. Ringhof S, Stein T. Biomechanical assessment of dynamic balance: specificity of different balance tests. Hum Mov Sci. 2018;58:140-7. https://doi.org/10. 1016/j.humov.2018.02.004.

81. Schwenk M, Schmidt M, Pfisterer M, Oster P, Hauer K. Rollator use adversely impacts on assessment of gait and mobility during geriatric rehabilitation. J Rehabil Med. 2011;43:424-9. https://doi.org/10.2340/16501977-0791.

82. Blankevoort CG, van Heuvelen MJG, Boersma F, Luning H, de Jong J, Scherder EJA. Review of effects of physical activity on strength, balance, mobility and ADL performance in elderly subjects with dementia. Dement Geriatr Cogn Disord. 2010;30:392-402. https://doi.org/10.1159/000321357.
83. Brett L, Traynor V, Stapley P. Effects of physical exercise on health and wellbeing of individuals living with a dementia in nursing homes: a systematic review. J Am Med Dir Assoc. 2016;17:104-16. https://doi.org/10.1016/j.jamda. 2015.08.016.

84. Lam FMH, Huang M-Z, Liao L-R, Chung RCK, Kwok TCY, Pang MYC. Physical exercise improves strength, balance, mobility, and endurance in people with cognitive impairment and dementia: a systematic review. J Physiother. 2018;64:4-15. https://doi.org/10.1016/j.jphys.2017.12.001.

85. Pitkälä KH, Savikko N, Pöysti MM, Strandberg TE, Laakkonen M-L. Efficacy of physical exercise intervention on mobility and physical functioning in older people with dementia: a systematic review. Exp Gerontol. 2013;48:85-93. https://doi.org/10.1016/j.exger.2012.08.008.

86. Scharpf A, Servay S, Woll A. Auswirkungen von körperlicher Aktivität auf demenzielle Erkrankungen. Sportwiss. 2013;43:166-80. https://doi.org/10. 1007/s12662-013-0295-7.

87. Trautwein S, Barisch-Fritz B, Scharpf A, Bossers WJR, Meinzer M, Steib S, et al. Recommendations for assessing motor performance in individuals with dementia: suggestions of an expert panel - a qualitative approach. Eur Rev Aging Phys Act. 2019;16:5. https://doi.org/10.1186/s11556-019-0212-7.

\section{Publisher's Note}

Springer Nature remains neutral with regard to jurisdictional claims in published maps and institutional affiliations.
Ready to submit your research? Choose BMC and benefit from:

- fast, convenient online submission

- thorough peer review by experienced researchers in your field

- rapid publication on acceptance

- support for research data, including large and complex data types

- gold Open Access which fosters wider collaboration and increased citations

- maximum visibility for your research: over $100 \mathrm{M}$ website views per year

At BMC, research is always in progress.

Learn more biomedcentral.com/submissions 